Article

\title{
Multi-Loop Model Reference Proportional Integral Derivative Controls: Design and Performance Evaluations
}

\author{
Baris Baykant Alagoz ${ }^{1}\left(\mathbb{D}\right.$, Aleksei Tepljakov ${ }^{2, *}$, Eduard Petlenkov ${ }^{2} \mathbb{C}$ and Celaleddin Yeroglu ${ }^{2} \mathbb{C}$ \\ 1 Department of Computer Engineering, Inonu University, 44000 Malatya, Turkey; \\ baykant.alagoz@inonu.edu.tr \\ 2 Department of Computer Systems, Tallinn University of Technology, 12616 Tallinn, Estonia; \\ eduard.petlenkov@taltech.ee (E.P.); c.yeroglu@inonu.edu.tr (C.Y.) \\ * Correspondence: aleksei.tepljakov@taltech.ee; Tel.: +90-555-273-70
}

Received: 27 December 2019; Accepted: 10 February 2020; Published: 13 February 2020

\begin{abstract}
Due to unpredictable and fluctuating conditions in real-world control system applications, disturbance rejection is a substantial factor in robust control performance. The inherent disturbance rejection capacity of classical closed loop control systems is limited, and an increase in disturbance rejection performance of single-loop control systems affects the set-point control performance. Multi-loop control structures, which involve model reference control loops, can enhance the inherent disturbance rejection capacity of classical control loops without degrading set-point control performance; while the classical closed Proportional Integral Derivative (PID) control loop deals with stability and set-point control, the additional model reference control loop performs disturbance rejection control. This adaptive disturbance rejection, which does not influence set-point control performance, is achieved by selecting reference models as transfer functions of real control systems. This study investigates six types of multi-loop model reference (ML-MR) control structures for PID control loops and presents straightforward design schemes to enhance the disturbance rejection control performance of existing PID control loops. For this purpose, linear and non-linear ML-MR control structures are introduced, and their control performance improvements and certain inherent drawbacks of these structures are discussed. Design examples demonstrate the benefits of the ML-MR control structures for disturbance rejection performance improvement of PID control loops without severely deteriorating their set-point performance.
\end{abstract}

Keywords: multi-loop model reference control; PID controllers; disturbance rejection control

\section{Introduction}

Control systems encounter unpredictable disturbances in real-world control applications. In order to maintain optimal control performance, real control systems should be designed to be robust enough against environmental disturbances, which are mainly unpredictable in character. From a practical point of view, performance degradations of classical control systems are largely caused by their dependence on predetermined system models. In the controller design phase, classical PID controllers are tuned to produce optimal control of a predetermined nominal plant model, which is indeed an idealized representation of a real-world system. The mathematical models of real systems can maintain their validity for predefined operating conditions and ranges, and only observable dynamics of the systems in these range and conditions can be represented by system models. Hence, they omit some inaccessible system dynamics and unknown disturbances, which are not rigorously investigated in the modeling stage. To enhance the representative capability of mathematical modeling, it is well-known that non-linear modeling [1], fractional-order modeling [2], and stochastic modeling [3] methods are 
often used. However, nowadays, optimal PID tuning methods still rely on integer order dynamics and linear system design approaches for the sake of simplicity and practical effectiveness. Therefore, the coverage potential of these modeling types to represent real-world conditions is quite limited, while mathematical models can represent the behavior of real systems in some presumed idealized conditions.

In general, optimal controller design in classical control has been addressed using integer order plant models and linear dynamics. Consequently, the designed controller law becomes mathematically optimal only for those ideal and linearized models operating in numerical simulation environments. However, when they are implemented in real-world conditions, practitioners commonly encounter the fact that the calculated controller coefficients are not practically optimal for real processes and fine-tuning efforts are needed for these controllers to achieve acceptable control performance. Firstly, non-ideal realization of controller function inherently deteriorates control optimality. Secondly, a number of factors such as unmodelled dynamics, noise, environmental disturbance, ageing of system components, and parametric perturbations may have a significant influence on control performance. Therefore, it is obvious that model-based optimal controller design methods should adhere to robust control performance requirements in the controller design stage, including considerations of disturbance rejection performance and insensitivity to model parameter variation. The current study focuses on enhancement of the disturbance rejection performance of classical control loops. The disturbance model is assumed to be additive input disturbance that has unknown characteristics. The disturbance rejection capacity of a classical negative feedback PID control loop is inherently bound [4]. A feasible solution to increase disturbance rejection performance beyond the inherent bounds of a PID control loop is by using a multi-loop control structure [5]. The findings of the current study suggest that a multi-loop model reference (ML-MR) adaptive control structure could be an effective solution to improve the disturbance rejection performance of classical PID control loops.

Due to a performance tradeoff between disturbance rejection control and set-point control performances [4], increasing the disturbance rejection control performance can severely deteriorate the set-point performance of classical PID control loops. A primary motivation of this study is the improvement of disturbance rejection control performance without effecting the set-point performance of existing closed loop PID control systems.

ML-MR control structures are commonly composed of two hieratical loops. These are the reference model loop, which describes the desired response of a system, and the control loop, which deals with the stability of control systems. The control loop is the primary loop that maintains the stability of the system. The secondary loop is the reference model loop, also called the adaptation loop, which handles the preservation of the desirable control performance of the system, which is described by the reference model response. When a disturbance acts on the control loop, the adaptation loop contributes to the control efforts for the reduction of the negative effects of disturbances on system outputs, thus restoring control performance by means of reference responses that are generated by the reference model.

In literature, numerous ML-MR control structures have been proposed and their improvements in terms of control performance have been demonstrated. In former works, direct model reference adaptive control (MRAC) structures have been addressed [5-10]. Direct MRAC structures perform online self-tuning of controller parameters according to a predefined reference model [5-8]. Updating controller parameters in control action, known as online controller tuning, may lead to short-term instability and control performance degradations. Therefore, the stability and robust control performance of MRAC structures in real conditions remain questionable and direct MRAC control methodologies have not been accepted or adopted by practitioners [8]. The industrial applications of the MRAC method have remained limited in comparison to the widespread adoption of classical PID control loops. PID control loops have been used as industrial standard control systems because of their well-established design scheme. The simple structure and reliability of PID control law, which facilitates realization in real systems, are prominent in industrial control applications. Barkana has addressed performance and stability issues of direct MRAC methods and presented a stable direct MRAC methodology according to passivity conditions [8]. However, to avoid online controller parameter updates in control action, 
one branch of ML-MR control research studies aims to combine a classical PID control system with reference model control in a hierarchical multi-loop manner in order to benefit from the stability and set-point performance of the classical control loop and the robust control performance improvements of the model reference control (MRC) system.

The topic of multi-loop control involves a wide variety of control systems that employ multi-loop control structures, including cascaded control loops [11,12], multi-input multi-output control systems [13], use of additional control loops for performance improvements [14,15], direct model reference control based on output matching $[16,17]$, direct model reference adaptive control with online controller tuning [5-10], model reference adaptive control based on Massachusetts Institute of Technology (MIT) rule [18-23], backstepping-based adaptive PID control [24], and hierarchical ML-MR adaptive control [25-28]. The current study investigates several configurations of hierarchical ML-MR adaptive control structures.

Hierarchical ML-MR adaptive PID control has been investigated, and certain benefits of this structure related to fault tolerance [25] and disturbance rejection [26] have been discussed. This structure can be easily applied to the existing closed loop PID control systems, thus transforming the PID control systems into model reference adaptive PID control systems. This presents a significant potential to improve the robust control performance of industrial PID control systems without largely modifying the existing PID loops. Although a growing research trend has been initiated for the use of ML-MR adaptive PID structures to improve the control performance of the closed loop PID control family [25-27], this control structure is still in the early stages of development. There is a need for further research efforts to establish design methodologies and reveal some practical benefits and drawbacks. Therefore, this study aims to investigate the benefits and drawbacks of ML-MR PID-MIT control $[25,28]$ and addresses design and performance improvement problems in ML-MR structures. To this end, this paper revisits recent multi-loop model reference adaptive PID control techniques, and analytical tuning and performance issues are considered. In analytical optimal tuning of PID controllers, we adopt the integral time absolute error (ITAE) design rule that was proposed by Tavakoli et al. for control of the first order plus dead time dynamic systems. This design rule is based on dimensional analysis, which has been widely utilized to solve complex high-dimensional problems by reducing the number of variables to an essential set [29]. The main reason to use the Tavakoli-Tavakoli ITAE design rule for optimal tuning of PID control loops is that the method limits overshoot with faster establishment of set-point control in time delay systems [29].

The organization of this paper is given as follows. Section 2 details the mathematical background of the multi-loop model reference PID-MIT (ML-MR PID-MIT) control, and presents analytical equations for theoretical background and analytical tuning. After discussing some inherent drawbacks of ML-MR PID-MIT structures (e.g., nonlinearity and amplitude dependence of disturbance rejection performance), several ML-MR control structures are investigated to resolve these drawbacks in the following sections. In these sections, two types of hierarchical ML-MR control structures are considered.

(i) Reference modeling of closed loop control loops: These types of MRC structures use a reference model to describe the desired response of closed loop control systems. Section 3 presents a multi-loop model reference PID-MIT control with controller gain modification (ML-MR PID-MIT-CGM) that allows dynamic disturbance rejection via adaptively adjusting Reference to Disturbance Ratio (RDR) performance. To deal with the drawbacks of nonlinearity from the MIT rule, a multi-loop model reference PID integral (ML-MR PID-I) control, which performs a linear adaptation rule, is introduced in this section. Section 4 introduces a multi-loop model reference PID-IM control structure (ML-MR PID-IM) that is proposed as an internal model linear adaptation rule to implement an internal model control (IMC) approach.

(ii) Reference modeling of plants: These types of MRC structures use a reference model to describe desired responses of a process or a plant function. Section 5 presents the multi-loop model reference MIT-PID control, which employs the model reference control rule only for plant function adaptation (ML-MR MIT-PID-PFA) in contrast to ML-MR MIT-PID in Section 2. The main advantages of this type 
of structure come from the fact that all adaptation efforts aim for approximation of plant or process models compared to the reference model. This property is advantageous for time delay systems. Section 6 presents a multi-loop model reference PID-PID control with plant function adaptation (ML-MR PID-PID-PFA). This structure employs a linear adaptation rule for plant function and it can improve control performance for time delay systems.

Illustrative design examples cover control the problems of an automatic voltage regulator model, the liquid level of reboilers, large time delay experimental process models, and Twin-Rotor Multi-input multi-output System (TRMS) experimental setup.

\section{Multi-Loop Model Reference PID-MIT (ML-MR PID-MIT) Control: Analytical Tuning and Nonlinearity}

The ML-MR PID-MIT control structure has been proposed to enhance the robust control performance of existing closed loop control systems. Its application has been discussed in several works [25-28]. This structure is essentially composed of two hierarchical control loops: the inner loop is a classical PID control loop that is a stable and well-tuned control loop, while the outer loop is a adaptation loop that performs the MIT rule. The outer loop is appended to the inner loop to enhance the robust control performance of the inner loop according to regulation of a reference model. Figure 1 shows a block diagram of the ML-MR PID-MIT control structure. It has two error signals per loop:

(i) Control error: When the controller of the inner loop is tuned properly, the value of the control error, which is written as $e_{c}=u_{r}-y$, moves to zero, and thus ensures the control system output settles to the reference input (the signal $u_{r}$ is the modified reference input controlled by the MIT rule and $y$ is the system output).

(ii) Model error: Discrepancy between the reference model output and controlled system output is defined as the model error, which is written as $e_{m}=y-y_{m}$ (the signal $y_{m}$ is the output of reference model). The objective of the outer loop is to shape the reference input signal $(r)$ such that the model error converges to zero. The convergence of the model error to zero implies that the inner loop resembles the response of the reference model $T_{m}(s)$. The reference model describes the desired response of the control system that forms the inner loop.

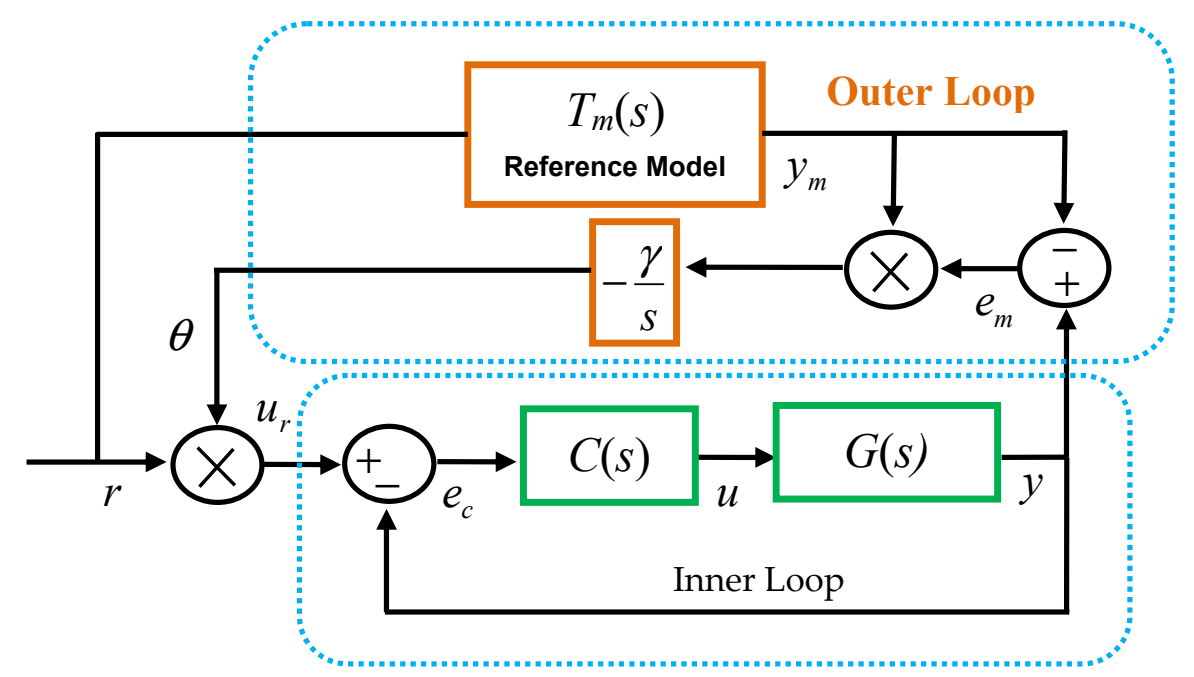

Figure 1. Block diagram of multi-loop model reference PID-MIT (ML-MR PID-MIT) control structure with input shaping.

Let us consider a PID control system as the inner control loop. The PID controllers are three-coefficient standard industrial controllers and optimal tuning of the PID coefficients $\left(k_{p}, k_{i}\right.$, 
$k_{d}$ ) enhances step performance by reducing overshoots and settling time. The transfer function of PID controllers is widely expressed in the parallel form as:

$$
C(s)=k_{p}+\frac{k_{i}}{s}+k_{d} s
$$

For a given plant (process) function $G(s)$, the transfer function of the inner loop can be written by:

$$
T(s)=\frac{C(s) G(s)}{1+C(s) G(s)}
$$

Previous studies on the ML-MR PID-MIT control structure aim to maintain the initial well-tuned control performance of an existing closed loop control system. For this reason, the reference model is taken as the transfer function of the inner loop; that is, $T_{m}(s)=T(s)$. Accordingly, any performance deterioration in the inner loop leads to an increase in model errors. The recovery of temporal performance deterioration is carried out by shaping the reference input of the inner loop via $u_{r}=\theta r$. The adaptation gain $\theta$ is determined according to the well-known MIT rule [21], which implements continuous gradient descent optimization of the cost function:

$$
J=\frac{1}{2} e_{m}^{2}
$$

The feed-forward MIT rule is expressed in the continuous domain as [18-21]:

$$
\frac{d \theta}{d t}=-\gamma \frac{d J}{d \theta}
$$

where the parameter $\gamma$ is the learning rate, which is an important parameter for the convergence speed and stability of the gradient descent optimization. Taking Laplace transform of Equation (4), one obtains:

$$
\theta=-\gamma \frac{1}{s} \frac{d J}{d \theta}=-\gamma \frac{1}{s} e_{m} \frac{d e_{m}}{d \theta}
$$

To find the sensitivity derivative $\frac{d e_{m}}{d \theta}$, the model error is written in the form of $e_{m}=y-y_{m}=$ $T(s) \operatorname{\theta r}-T_{m}(s) r$. Then, the sensitivity derivative of the system is obtained:

$$
\frac{d e_{m}}{d \theta}=T(s) r
$$

Here, one can substitute the reference input with $r=y_{m} / T_{m}(s)$ in Equation (6) and rearrange the sensitivity derivative as:

$$
\frac{d e_{m}}{d \theta}=\frac{T(s)}{T_{m}(s)} y_{m}
$$

By using it in Equation (5), the MIT rule for the update of the adaptation coefficient $\theta$ to minimize cost function $J$ is obtained as $[25,26,28]$ :

$$
\theta=-\gamma \frac{1}{s}\left(\frac{T(s)}{T_{m}(s)} y_{m} e_{m}\right)
$$

When one configures $T_{m}(s)=T(s)$, the adaptation gain $\theta$ is simplified to:

$$
\theta=-\gamma \frac{1}{s} y_{m} e_{m}
$$

which is implemented in the outer loop in Figure $1[25,26,28]$.

Application of ML-MR PID-MIT control for the existing control loop was defined as follows [25,26,28]: 
Step 1: Identify the transfer function model of the existing closed loop PID control system by means of closed loop model identification methods and use this transfer function as a reference model.

Step 2: Enclose the existing closed loop control system via the outer loop. The outer loop employs Equation (9) and the reference model control.

To create a design from scratch for a first order system model in the form of $G(s)=\frac{K}{\tau s+1} e^{-L s}$, an analytical tuning scheme for the ML-MR PID-MIT control structure can be proposed as follows:

Step 1: Design a closed loop PID control system according to the Tavakoli-Tavakoli PID tuning rule that can be rearranged for a standard PID controller function in parallel form as follows [29]:

$$
k_{p}=(1 / K) \frac{0.8}{(L / \tau)+0.1}, k_{i}=k_{p} \frac{1}{(0.3 L+\tau)}, k_{d}=k_{p} \frac{0.06 L}{(L / \tau)+0.04}
$$

This optimal tuning rule implements ITAE and its control performance have been shown previously [29]. The optimal PID controller function of the inner loop can be written for the TavakoliTavakoli optimal PID tuning rule as:

$$
C(s)=(1 / K) \frac{0.8}{(L / \tau)+0.1}\left(1+\frac{1}{(0.3 L+\tau) s}+\frac{0.06 L}{(L / \tau)+0.04} s\right)
$$

Step 2: The reference model is a theoretical model that represents the optimally tuned closed loop control system. Therefore, the transfer function of reference model can be expressed as:

$$
T_{m}(s)=\frac{C(s) G(s)}{1+C(s) G(s)}
$$

In MATLAB and Simulink control system simulations, we implemented this reference model as a numerical model of the PID control loop, as illustrated in the diagram in Figure 2.

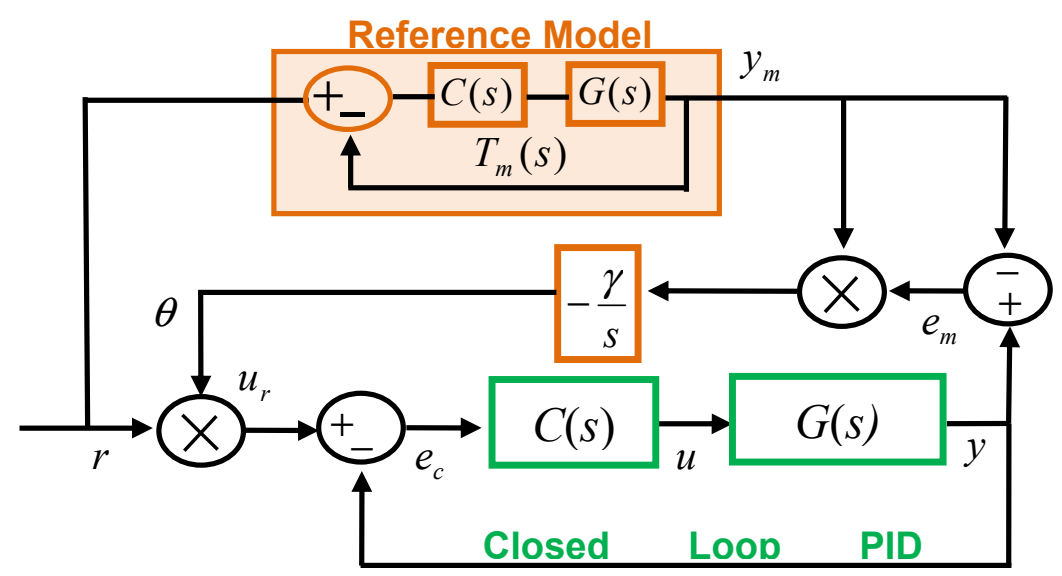

Figure 2. Block diagram of the ML-MR PID-MIT control structure and a solution for numerical realization of reference models.

Step 3: Implement the ML-MR PID-MIT control structure as shown in Figure 2. The learning rate $\gamma=\left|k_{i}\right|$ can be selected to comply with integral dynamics of the PID control loop.

It is important to note two characteristic features of this structure. Firstly, the performance of the ML-MR PID-MIT control structure is sensitive to the time delay component of the system. As the time delay of the system increases, the adaptation performance of the MIT rule decreases because large delay in the system response easily misleads gradient descent optimization of the MIT rule. Figure 3 shows this effect for the ML-MR PID-MIT control structure design for control of the plant functions with zero time delay $\left(G_{1}(s)=\frac{2}{s+1}\right)$ and $0.3 \mathrm{~s}$ time delay $\left(G_{2}(s)=\frac{2}{s+1} e^{-0.3 s}\right)$ [29]. The system responses in the figure demonstrate that the ML-MR PID-MIT control structure can significantly improve the 
disturbance rejection performance of classical PID control for zero delay systems. However, when the time delay of the plant increases, improvement in disturbance rejection control decreases.

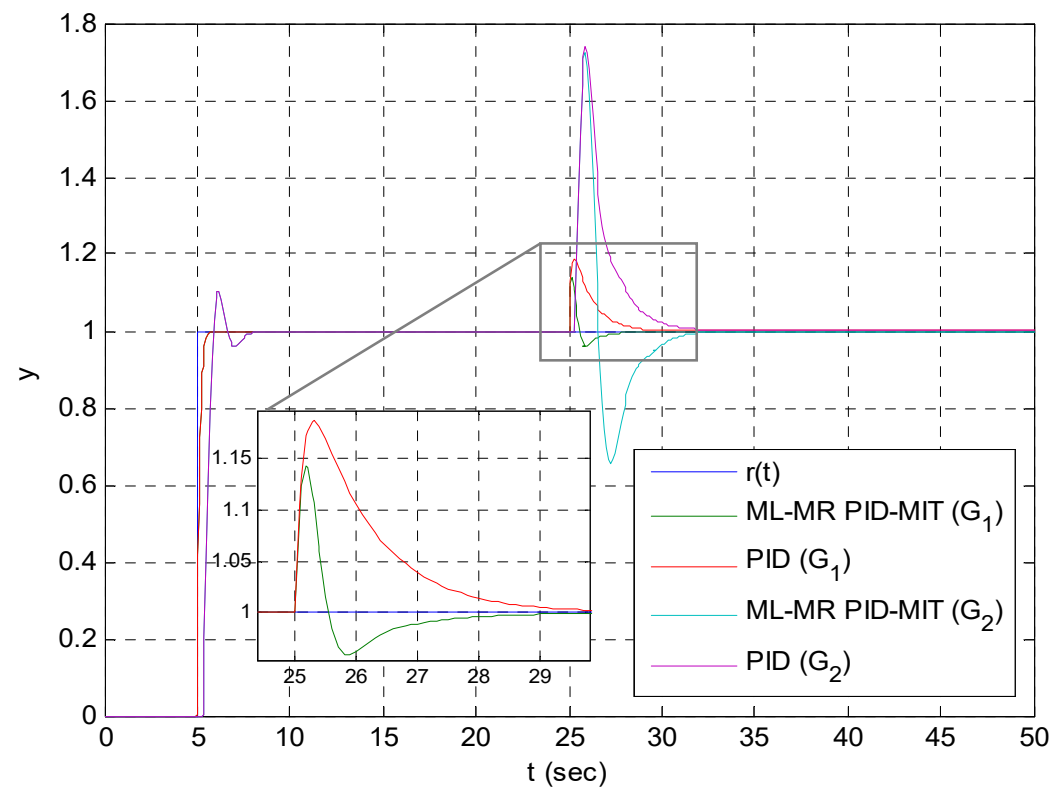

Figure 3. Comparison of disturbance rejection performance of ML-MR PID-MIT control and classical PID control for $G_{1}(s)$ and $G_{2}(s)$ plants.

Secondly, due to multiplication terms $u_{r}=\theta r$ and $y_{m} e_{m}$, the ML-MR PID-MIT control structure presents a nonlinear control characteristic. The origin of the nonlinearity in the adaptation gain $\theta$ is the nonlinear cost function, which involves the square of model error in Equation (3). This yields a nonlinear term $y_{m} e_{m}=y_{m}\left(y-y_{m}\right)=y_{m} y-y_{m}^{2}$. Therefore, ML-MR PID-MIT control can exhibit some characteristic properties that do not appear in linear control systems. A noteworthy property is that the time response of the PID-MIT control shows amplitude dependence. In the case of reference input $r(t)=0$, this results in $y_{m} \cong 0$; in this case, the outer loop is not functional, so that $y_{m} e_{m}=0$ in Equation (9). As the amplitude of the reference input signal $r(t)$ grows, the amplitude of $y_{m}$ increases, and this effect indeed amplifies the model error due to the term of $y_{m} e_{m}$. Accordingly, this makes the MIT rule of the outer loop more sensitive to model errors and the system responds more effectively in cases where the model mismatches between reference model and control loops. To demonstrate this effect, an illustrative example for control of the plant function $G(s)=\frac{2}{s+1}$ is carried out.

Figure 4 shows time response ML-MR PID-MIT control structure that is designed for plant function $G(s)=\frac{2}{s+1}$ [29]. The learning rate $\gamma$ is set to -4 , and the PID controller is configured to $k_{p}=4, k_{i}=4$, and $k_{d}=0$ according to the Tavakoli-Tavakoli PID tuning rule in this simulation. When the amplitude of $r(t)$ moves to one $(r(t)=1)$, the ML-MR PID-MIT control structure becomes more effective and contributes to the disturbance rejection control performance of the inner loop (classical PID control loop). However, when the amplitude of $r(t)$ moves to zero $(r(t)=0)$, the ML-MR PID-MIT control structure is not functional, and its disturbance rejection control performance becomes the same as the disturbance rejection performance of the classical PID controller. The overlapping of time responses at set-point zero in the figure apparently confirms this effect. 

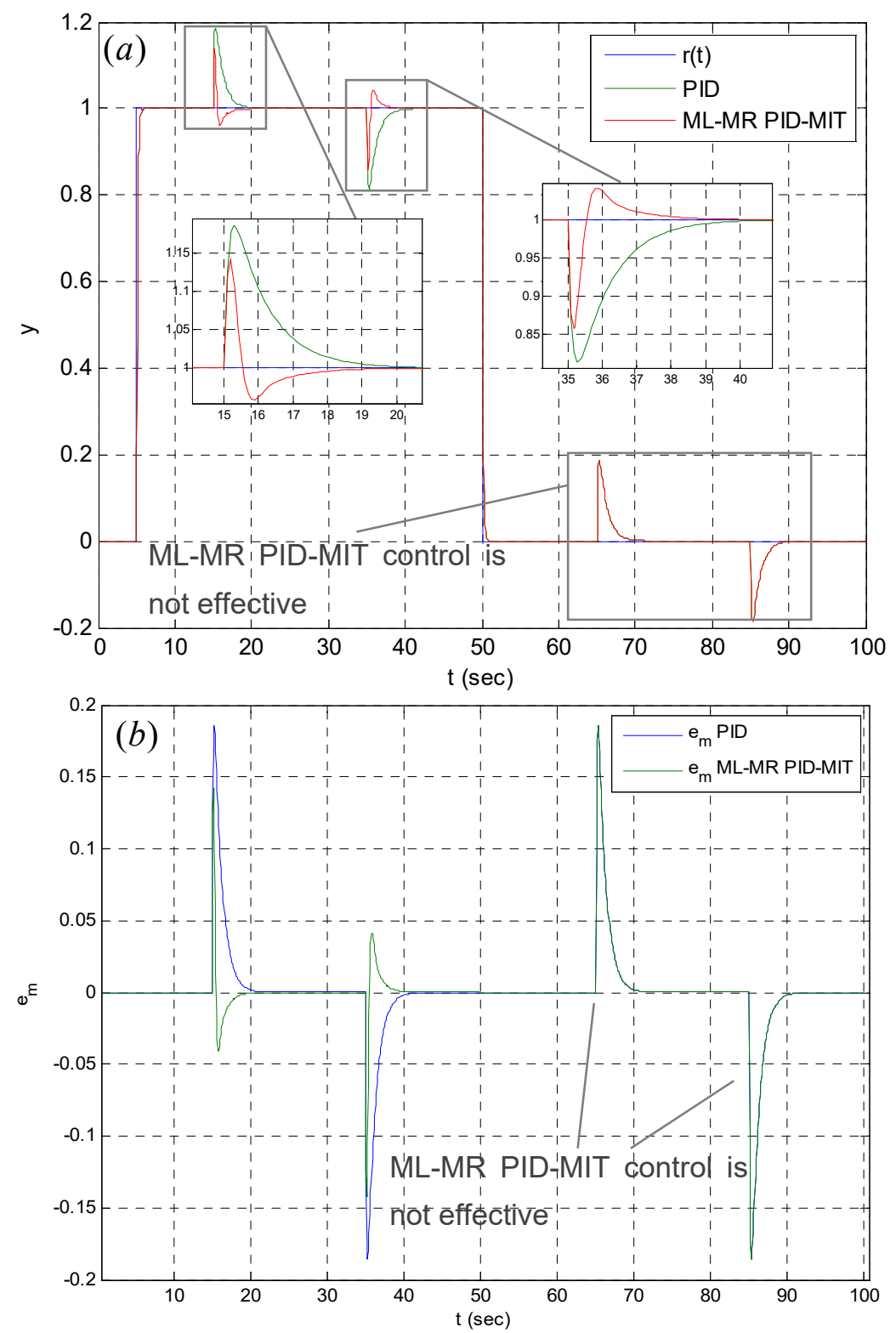

Figure 4. Comparison of disturbance rejection performances of ML-MR PID-MIT control and classical PID control for the cases of $r(t)=1$ and $r(t)=0$. (a) Step response and (b) change of model error.

\section{Multi-Loop Model Reference PID-MIT with Controller Gain Modification (ML-MR PID-MIT-CGM): Adaptive RDR Adjustment for Dynamic Disturbance Rejection and the Linear Adaptation Rule}

The disturbance rejection capacity of a closed loop control system can be expressed with the reference to disturbance ratio (RDR) spectrum, which presents a measure for the additive input disturbance rejection performance of a control system for each frequency component [4,30]. The analysis demonstrated that the RDR performance of a closed loop control system depends on the spectral power density of the controller function [4]. The RDR spectrum of the PID controller is expressed in the form of $[4]$ :

$$
R D R_{P I D}(\omega)=k_{p}^{2}+\left(k_{d} \omega-\frac{k_{i}}{\omega}\right)^{2}
$$

The increase of low frequency RDR values improves the disturbance rejection control for low frequency and steady disturbance signals. An increase in high frequency RDR values improves the transient disturbance rejection performance. However, as mentioned previously, the tradeoff between 
disturbance rejection control and set-point control performance bounds the RDR performance of the closed loop PID control systems [4]. A large increase in RDR values deteriorates the step response performance; this results in very high overshoots and consecutive ripples, which delay the convergence of the controlled system output to the set-point. Further increases in RDR values finally lead to instability of the control systems [4]. Therefore, the unstable state of the closed loop control systems limits the improvement of their RDR performances. A solution for this problem is adaptive adjustment of RDR performance; RDR performance should be increased for disturbance incidents in the inner loop. In order to adjust the RDR performance of the PID control loop adaptively, a variable gain PID controller is proposed by multiplying the PID controller function with the adaptation coefficient $\theta$. This modified PID controller function allows adjustment of the DC gain of the classical PID controller by means of the coefficient $\theta$. The transfer function of this controller can be expressed as:

$$
C_{\theta}(s)=\theta\left(k_{p}+\frac{k_{i}}{s}+k_{d} s\right)
$$

The RDR spectrum of this RDR-adjustable PID controller can be obtained as:

$$
\operatorname{RDR}_{P I D \theta}(\omega)=\theta^{2}\left(k_{p}^{2}+\left(k_{d} \omega-\frac{k_{i}}{\omega}\right)^{2}\right)
$$

This expression of the RDR spectrum apparently shows that the proposed variable gain PID controller $C_{\theta}(s)$ allows modification of the RDR spectrum via adaptation gain $\theta$. This enables adaptive adjustment of the disturbance rejection performance of the inner loop by the outer loop. Since the gain $\theta$ amplifies the control error inside the inner loop, the value of the adaptation gain $\theta$ should be configured to remain positive, and hence is written by:

$$
\theta=\left|\gamma \frac{1}{s} y_{m} e_{m}\right|
$$

Figure 5 shows the RDR spectrum of the controller $C_{\theta}(s)$ for several values of the gain $\theta$. This figure reveals that the proposed variable gain PID controller $C_{\theta}(s)$ enables modification of the RDR spectrum via the gain $\theta$, and this asset allows adaptive adjustment of disturbance rejection performance in the control loop. For $\theta=1$, the $C_{\theta}(s)$ controller exhibits disturbance rejection performance equal to the performance of the classical PID controllers. When $\theta>1$, the disturbance rejection performance of $C_{\theta}(s)$ increases, as illustrated in the RDR spectrum in Figure 5. It should be noted that the variable gain PID control may cause instability, particularly for time delay systems, because higher values of the gain $\theta$ result in greater amplification of the integral gain, which may cause instability in the control systems as a consequence of accumulation of integral element control errors during the delay period of the system response. This limits the application domain of the proposed method to stable and almost-zero time delay plant functions.

The application steps for ML-MR PID-MIT with controller gain modification (ML-MR PID-MIT-CGM) for existing control loops can be given as follows:

Step 1: Obtain a transfer function model of an optimal control loop and use this transfer function as a reference model of the MRC loop $\left(T_{m}(s)=T_{1}(s)\right)$.

Step 2: Enclose the existing closed loop control system by the outer loop, as shown in Figure 6. Apply the MIT rule for controller gain modification of the closed loop PID control system to adjust the RDR.

To show an application of the ML-MR PID-MIT-CGM control structure, an Automatic Voltage Regulator (AVR) control example is presented in this section. Figure 7 shows a block diagram of the proposed ML-MR PID-MIT-CGM control structure for the AVR control example. The learning rate $\gamma$ is set to 10 . 


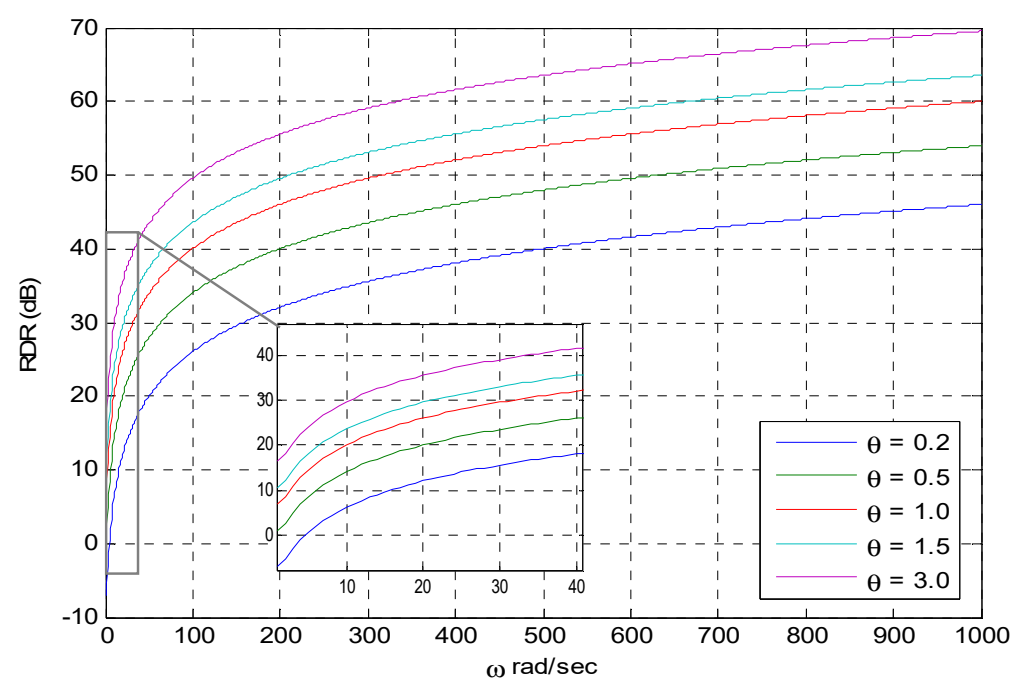

Figure 5. RDR spectra of $C_{\theta}(s)$ controllers for several $\theta$ values. This figure reveals the adjustment of RDR performance according to several $\theta$ configurations.

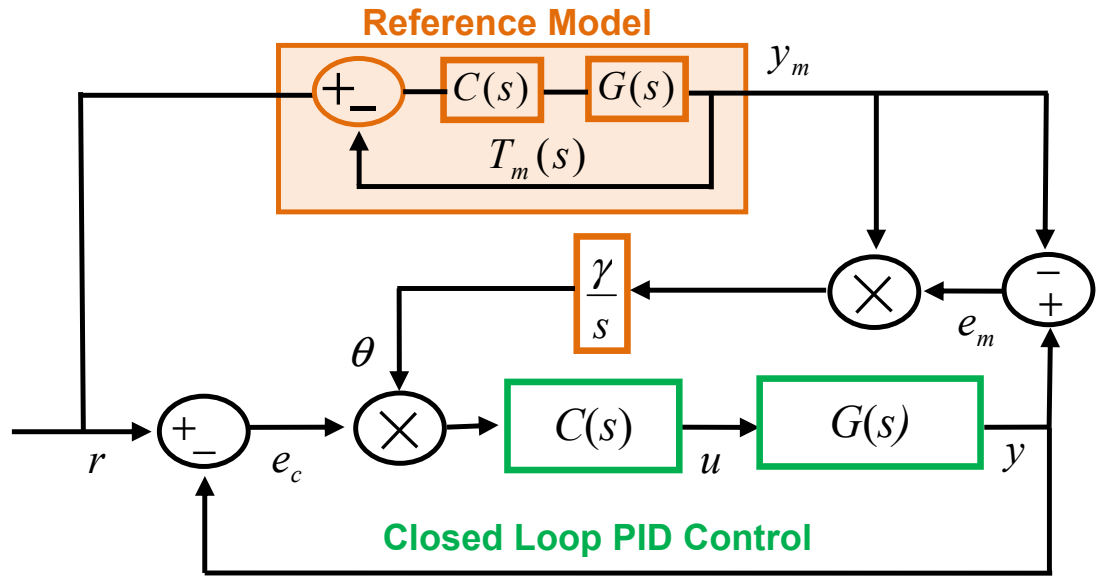

Figure 6. Block diagram of the ML-MR PID-MIT with controller gain modification (ML-MR PID-MIT-CGM) control structure and numerical realization of the reference model.

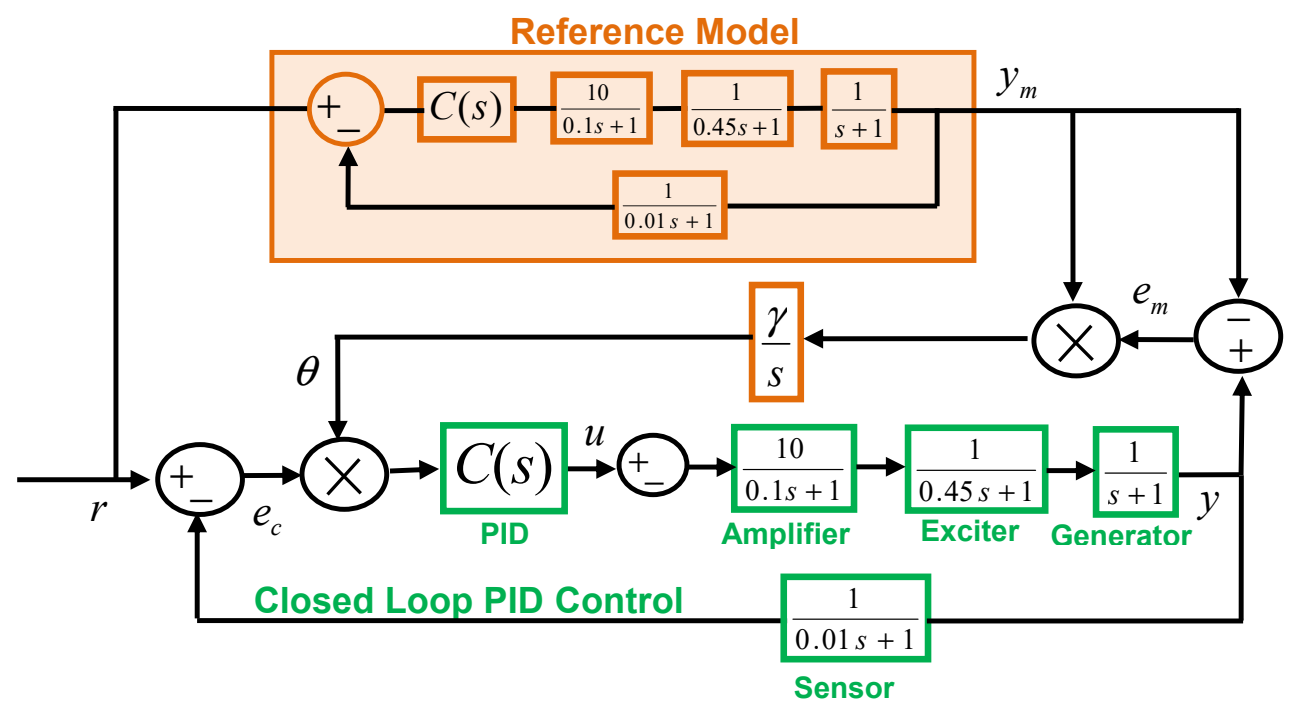

Figure 7. ML-MR PID-MIT-CGM control structure with control gain shaping that is implemented for improvement of the disturbance rejection performance of an optimal closed loop PID control of an AVR model. 
Bendjeghaba et al. implemented an improved harmony search algorithm for optimal tuning of closed loop PID control of an AVR model. The optimal PID controller was obtained by Bendjeghaba et al. in [31] as:

$$
C(s)=0.6739+\frac{0.5076}{s}+0.2699 s
$$

We used this optimal PID controller for the AVR model in Figure 7. Figure 8a,b show responses of Bendjeghaba et al.'s optimal PID control loop and ML-MR PID-MIT-CGM control structure for square waveform input disturbances. The figure clearly shows disturbance rejection performance improvements for the ML-MR PID-MIT-CGM control at simulation times of 15 and $35 \mathrm{~s}$, where the set-point is equal to 1 . At these instances, the adaptation gain $\theta$ of the outer loop actively responds to step-up and step-down disturbances, and it increases the RDR performance of the inner loop by increasing $\theta$, as illustrated in Figure 9. This effect provides a better disturbance rejection control than the classical PID control loop. Due to the amplitude dependence of the MIT rule, contributions of the ML-MR PID-MIT-CGM control to the disturbance rejection performance begin to vanish in the vicinity of the set-point 0 . Consequently, the system can respond slightly better for step-up and step-down disturbances at the simulation times of 65 and $85 \mathrm{~s}$ compared to the optimal PID loop proposed by Bendjeghaba et al.
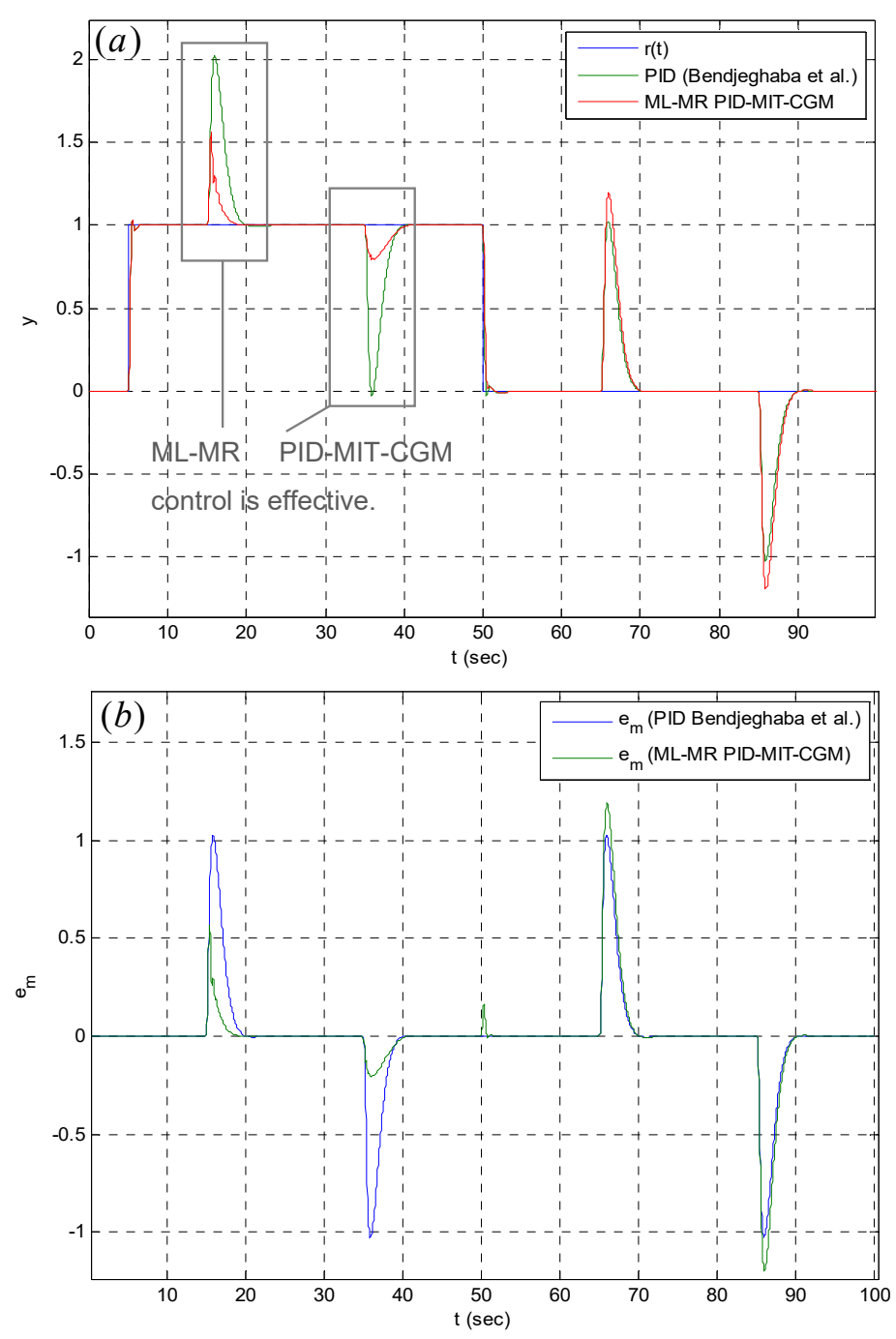

Figure 8. Improvement of the disturbance rejection performance of the classical optimal PID loop by the proposed ML-MR PID-MIT-CGM control structure for the AVR control system model. (a) Step response and (b) change of model error. 

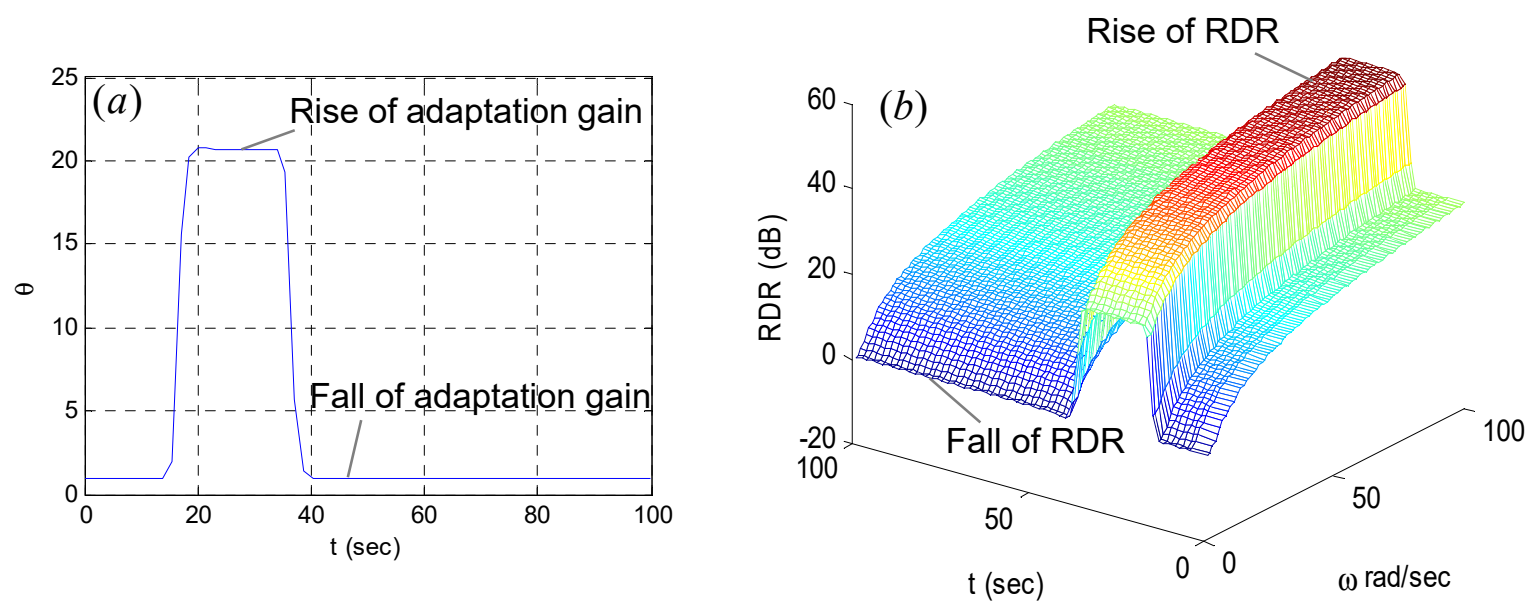

Figure 9. (a) Evolution of the adaptation gain $\theta$ of the ML-MR PID-MIT-CGM control structure. (b) Corresponding adaptive modification of the RDR spectrum of the $C_{\theta}(s)$ controller in order to reject disturbances.

Figure 9 describes how ML-MR PID-MIT-CGM control responds to disturbance incidents. For a disturbance incident, the gain $\theta$ increases, as shown in Figure 9b. This results in an increase of PID coefficients, and accordingly an increase in the RDR rates in the spectrum, as shown in Figure 9b. Therefore, the RDR performance of the optimal PID control loop of Bendjeghaba et al. is adaptively modified by adaptation gain $\theta$, while the disturbances affect the system response. This figure verifies an important asset of the proposed ML-MR PID-MIT-CGM control structure: It adaptively adjusts the disturbance rejection control performance and retains the set-point control performance of the optimal PID loops in absence of disturbance incidents. Thus, it overcomes the inherent shortcoming that is caused by the performance tradeoff between set-point control and disturbance rejection control. This property can significantly contribute to the practical robust control performance of classical control loops and can be viewed as an elegant solution for increasing the disturbance rejection performance without deteriorating the set-point performance of control systems. However, there will be a few drawbacks in the PID-MIT structures for linear control system applications. The nonlinearity of the MIT rule leads to dependence of disturbance rejection performance on the level of the set-point. Another drawback for linear control applications is that the performance of the PID-MIT-CGM control structure is very sensitive to the time delay of plants. A sufficiently large time delay may easily lead to instability in the systems.

As mentioned in the previous section, the MIT rule does not behave linearly due to the terms of $y_{m} e_{m}$. To eliminate this nonlinearity and to make structural contributions to the PID control loop independent of the level of signal amplitudes, one can update the term $y_{m} e_{m}$ as $c e_{m}$, where the constant $c \in R$ can be typically set to 1 . This modification implies that the sensitivity derivative is constant and can be shown as $\frac{d e_{m}}{d \theta}=c$. Hence, Equation (16) is simplified to an integral operator and the adaptation gain can be written as:

$$
\theta=\left|\gamma \frac{1}{s} e_{m}\right|
$$

Using this modified adaptation gain-where Equation (18) replaces the MIT rule in Equation (16) -produces a new ML-MR control structure, which is called multi-loop model reference PID integral (ML-MR PID-I) control. The ML-MR PID-I control structure behaves linearly and improves the disturbance rejection performance independent of the reference input amplitude. Results in Figure 10 demonstrate this effect. Although ML-MR PID-MIT-CGM is very effective around the unity set-point, its performance degrades at set-point 0 , although it is slightly better than the optimal PID loop proposed by Bendjeghaba et al. However, ML-MR PID-I control presents the same performance as ML-MR PID-MIT control at the unity set-point and can maintain this performance improvement at set-point 0 . Figure 11 validates this effect by showing the adaptive adjustment of the RDR spectrum at set-point 
levels 1 and 0 . As a consequence, for linear control systems with almost zero time delay, the proposed ML-MR PID-I control structure is capable of improving the disturbance rejection performance without deteriorating the set-point performance.
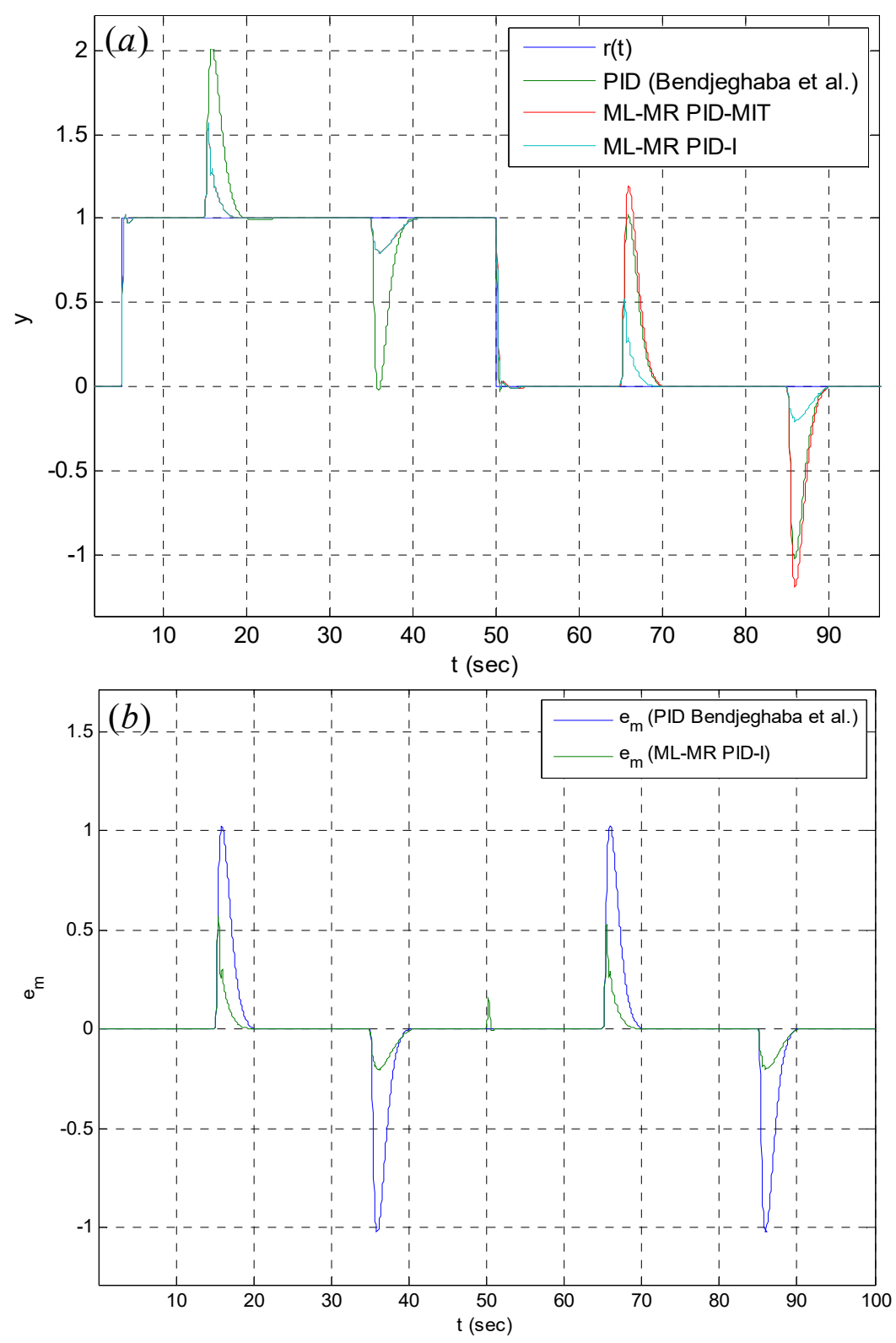

Figure 10. Improvement of the disturbance rejection performance of the classical optimal PID loop by the proposed ML-MR PID-I control structure for the model. (a) Step response and (b) change of model error.

The simulation results verify the effectiveness of the ML-MR control loops by showing that the ML-MR control has a performance capacity beyond the typical disturbance rejection of classical single-loop control loops. This is a very useful property that improves the robust control performance in the case of disturbance interference to the control system. The ML-MR PID-MIT-CGM control structure is more preferable for set-point control of nonlinear systems, where linear ML-MR control structures are not an effective solution. 

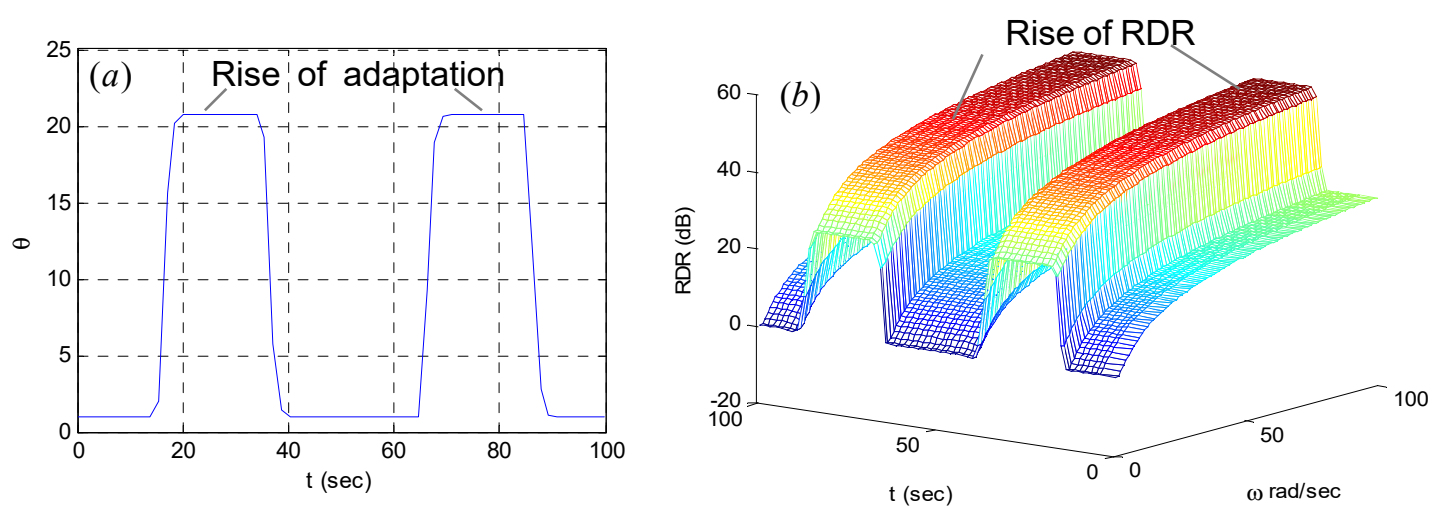

Figure 11. (a) Evolution of adaptation gain $\theta$ of the ML-MR PID-I control structure. (b) Adaptive modification of the RDR spectrum of the $C_{\theta}(s)$ controller for disturbance rejection.

\section{Multi-Loop Model Reference PID Internal Model (ML-MR PID-IM) Control Structure: Internal Model Linear Adaptation Rule}

Internal model control (IMC) relies on a mathematical model of a plant or process, which is referred to as an internal model and performs the control law according to this internal model. It was reported that IMC can be an effective solution for disturbance rejection and model perturbation problems [32]. In this section, we consider IMC to design a ML-MR control structure. Previously, an IMC-PID controller was proposed for improved disturbance rejection of time-delayed processes by using the reference model of the plant [14]. IMC law can be designed by manipulating transfer function models of the control system. If the internal model is an accurate enough representation of the system, the derived control law in the s-domain becomes valid for the real system.

In this section, we assume that the internal model is the PID control loop (the inner loop) and design an internal model PID controller, such that the outer loop can perform the IMC for the inner loop. Let us consider the multi-loop control structure that is depicted in Figure 1. To improve the disturbance rejection performance without influencing the control performance of the inner loop, the reference model, which is the internal model, is assumed to be the transfer function of the inner loop.

$$
T_{m}(s)=T_{1}(s)=\frac{C_{1}(s) G(s)}{1+C_{1}(s) G(s)}
$$

One can write the transfer function that involves the internal model controller loop $C_{2}(s)$ and the theoretical reference model $T_{1}(s)$ as:

$$
T_{2}(s)=\frac{C_{2}(s) T_{1}(s)}{1+C_{2}(s) T_{1}(s)}
$$

Our design objective is to find an internal model controller $C_{2}(s)$ so that the whole system behaves the same as the theoretical reference model $T_{m}(s)$, which represents an optimal design of the PID control loop. In order to achieve this objective, the transfer function condition $T_{2}(s)=T_{m}(s)$ should be satisfied. By using $T_{m}(s)=T_{1}(s)$ (Equation (19)), the transfer function condition becomes $T_{2}(s)=T_{m}(s)=T_{1}(s)$. It is used in Equation (20) to satisfy the transfer function condition, which gives:

$$
T_{1}(s)=\frac{C_{2}(s) T_{1}(s)}{1+C_{2}(s) T_{1}(s)}
$$

Then, the controller function $C_{2}(s)$ is written as:

$$
C_{2}(s)=\frac{1}{1-T_{1}(s)}
$$


By using Equation (19) in Equation (22), the $C_{2}(s)$ function is found:

$$
C_{2}(s)=C_{1}(s) G(s)+1=L(s)+1,
$$

where the $L(s)$ function is the open loop transfer function of the inner loop, which is expressed as $L(s)=C_{1}(s) G(s)$. In this form, the $C_{2}(s)$ function can be directly implementable. Application steps for existing control loops are summarized as follows:

Step 1: The transfer function model of an optimal control loop is obtained, and this transfer function is used as the reference model $\left(T_{m}(s)=T_{1}(s)\right)$.

Step 2: The optimal closed loop control system is enclosed by the outer loop, as shown in Figure 12.

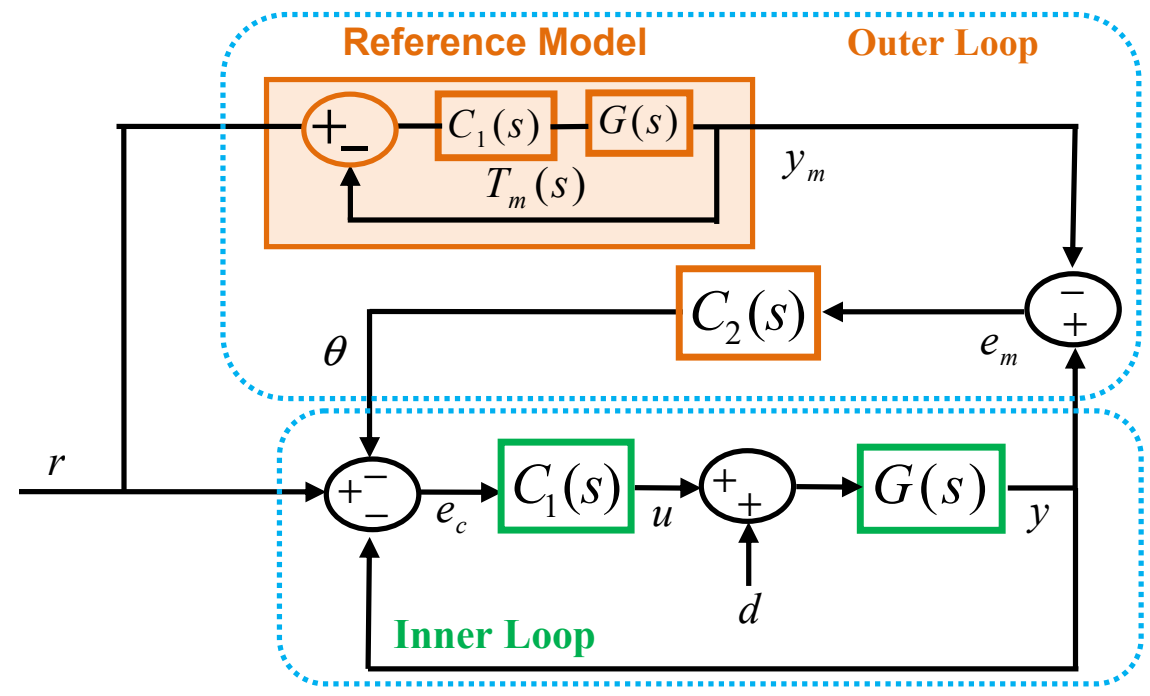

Figure 12. Block diagram of the ML-MR PID-IM control structure.

To demonstrate a possible application for the ML-MR PID-IM control, an example design for level control problem is presented. The plant model is an approximate model that represents the liquid level in the reboiler of the steam-heated distillation equipment [14,33]. The liquid level is controlled by adjusting the control valve on the steam line and the process model is given as $G(s)=\frac{-1.6(-0.5 s+1)}{3 s(s+1)}[14,33]$. The optimal PID controller for improved disturbance rejection was given as [33]:

$$
C_{1}(s)=-1.25-\frac{0.2358}{s}-1.8125 s
$$

The system model is unstable, and the controller function was obtained with negative coefficients. By considering Equation (23), the $C_{2}(s)$ function of the ML-MR PID-IM control structure is obtained as:

$$
C_{2}(s)=\left(-1.25-\frac{0.2358}{s}-1.8125 s\right) \frac{-1.6(-0.5 s+1)}{3 s(s+1)}+1
$$

We implemented the ML-MR PID-IM control structure for this system, as shown in Figure 13, and performed control simulations for square waveform input disturbance.

Figure 14 shows responses of the ML-MR PID-IM control structure and Chen et al.'s optimal PID control loop. Results in Figure 14 clearly demonstrate that the ML-MR PID-IM control structure improves the disturbance rejection performance of Chen et al.'s optimal PID control loop without influencing its set-point performance. In the absence of disturbance, responses of the ML-MR PID-IM control structure overlap with responses of the optimal PID control loop. The set-point performance of the ML-MR PID-IM control is almost the same as the performance of the optimal PID control loop proposed by Chen et al. This is an important advantage for robust control performance so that the proposed ML-MR control structures can improve the disturbance rejection while maintaining the 
set-point performance of the existing PID control loops. A limitation of the ML-MR PID-IM control is that it is not an effective solution for large time delay plant dynamics and inaccurate plant models. High time delays can severely affect the performance of the ML-MR PID-IM control scheme.

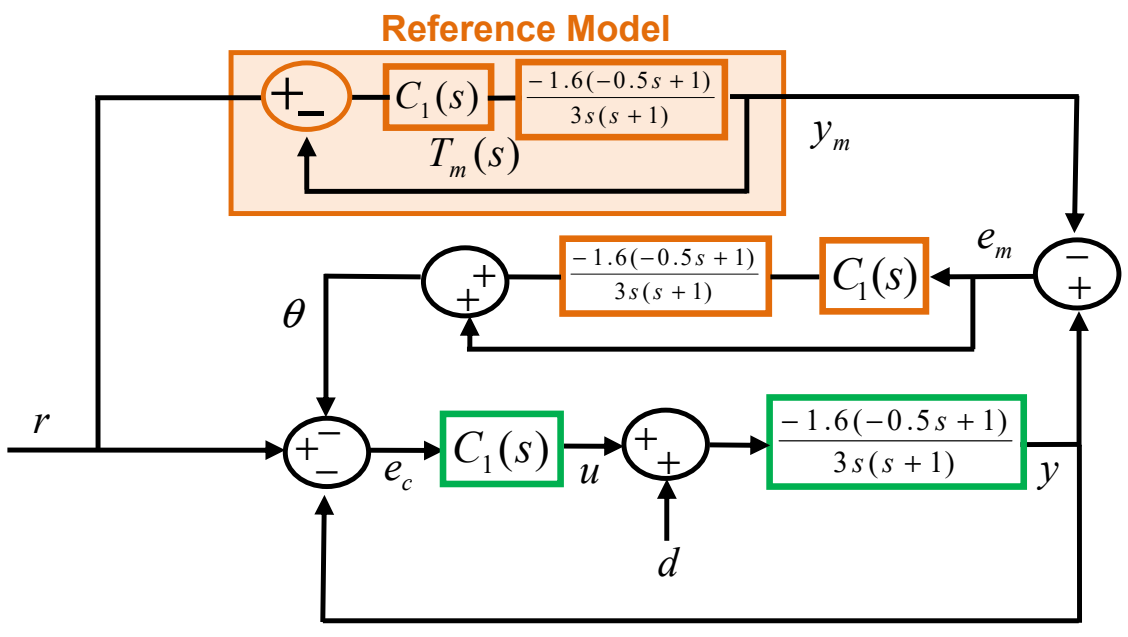

Figure 13. Implementation of the ML-MR PID-IM control structure for disturbance rejection control of the liquid level of the reboiler model.
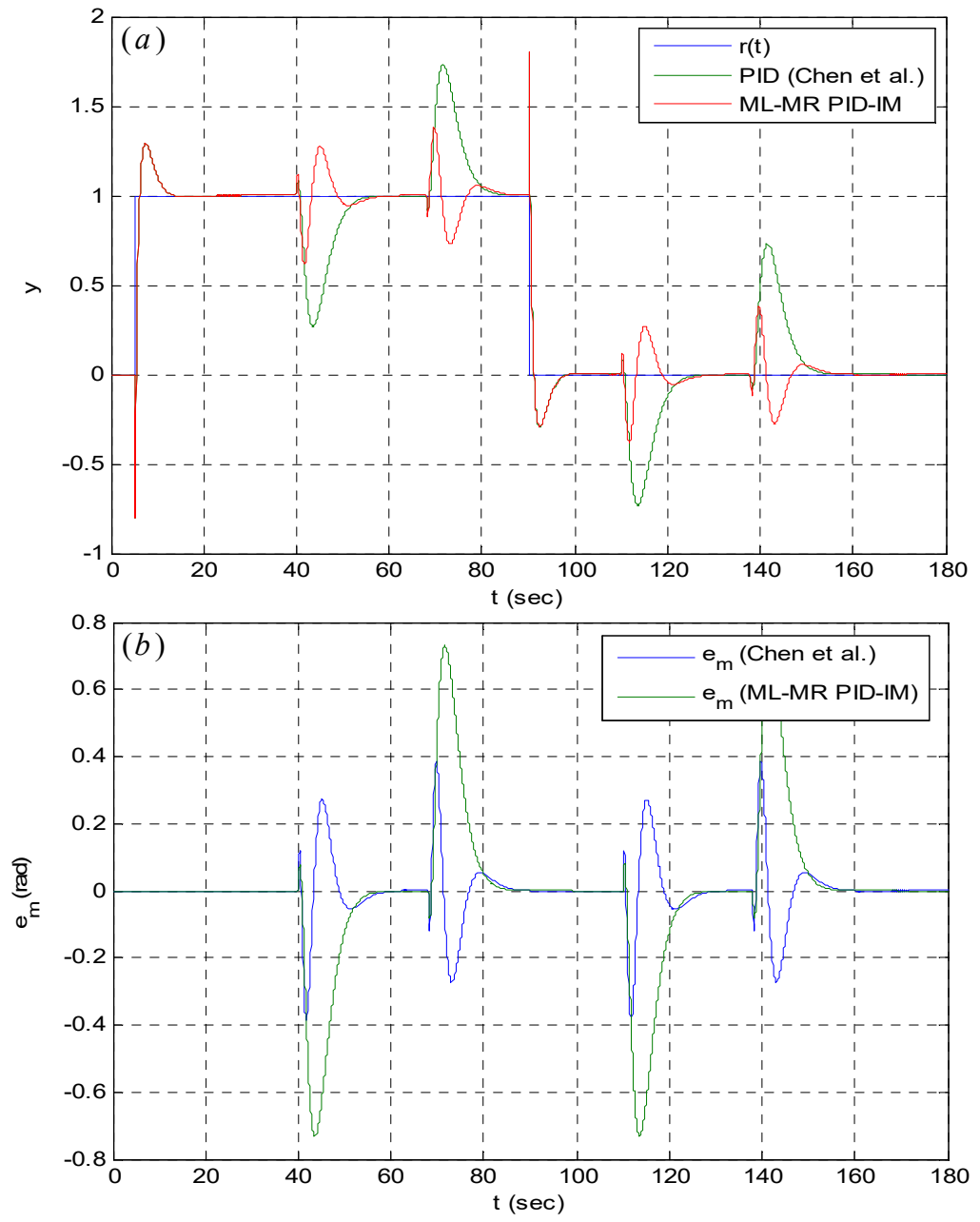

Figure 14. Improvement of the disturbance rejection performance of the classical optimal PID loop by the proposed ML-MR PID-IM control structure for liquid level control in the reboiler model. (a) Step response and (b) change of model error. 


\section{Multi-Loop Model Reference MIT-PID with Plant Function Adaptation (ML-MR MIT-PID-PFA): Nonlinear Adaptation Rule for Time-Delayed Systems}

To improve the disturbance rejection capacity of time delay systems, model reference control is adopted for plant functions in a similar manner to classical IMC [14]. Any mismatch in responses from the real system and mathematical models of plants is assumed to be disturbance and is rejected by contributions of the MIT rule. In this configuration, the MIT rule forms the inner loop. The outer loop deals with the stability and set-point control. Therefore, this type of ML-MR control structure can be more effective for time delay systems because the adaptation loop only handles model mismatches between real plants and their mathematical models.

Given a first order plus dead time plant function in the form of $G(s)=\frac{K}{\tau s+1} e^{-L s}$, an analytical design scheme for the ML-MR MIT-PID-PFA control structure can be suggested as follows.

Step 1: Identify a model of the plant function and take the reference model of the MRC loop as the plant function $\left(T_{m}(s)=G(s)\right)$.

Step 2: Enclose the MRC loop with an optimal closed loop PID controller loop, as shown in Figure 15. Use the Tavakoli-Tavakoli PID tuning rule, which is expressed by Equation (10), for optimal operation of the PID controller function $C(s)$.

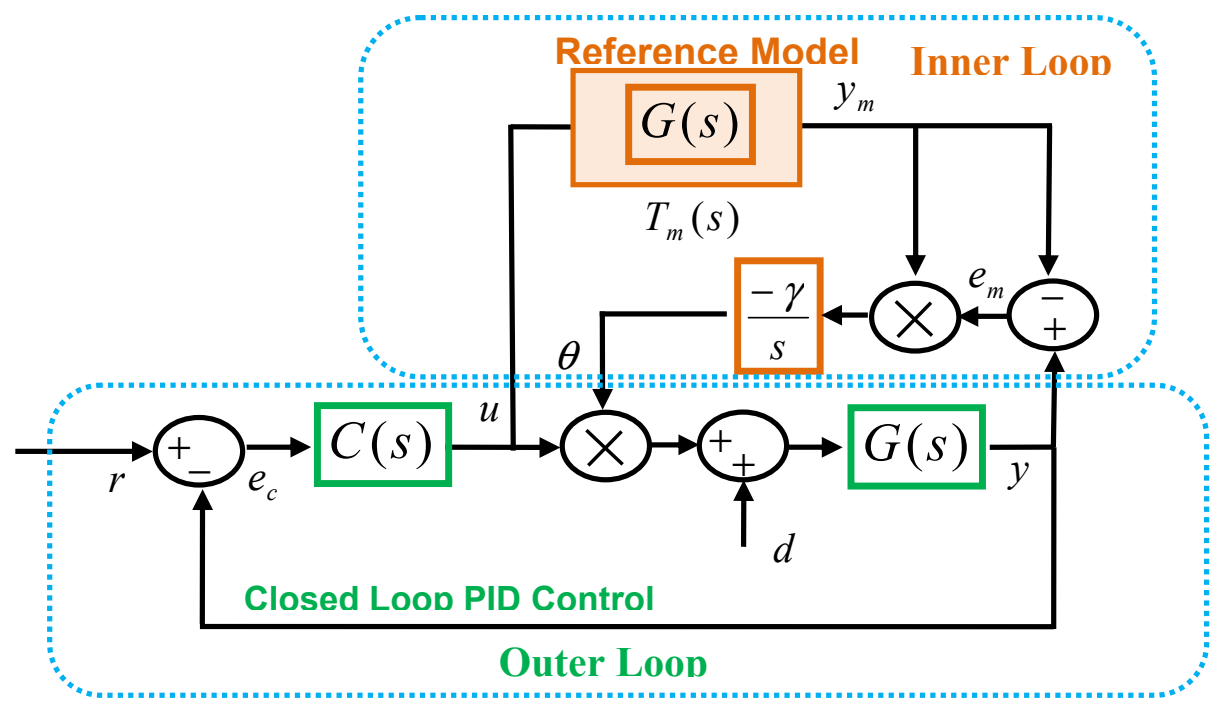

Figure 15. Block diagram of the ML-MR MIT-PID-PFA control structure.

An example design for the first order plus time delay plant model is given in the following section.

\section{Multi-Loop Model Reference PID-PID with Plant Function Adaptation (ML-MR PID-PID-PFA): Linear Adaptation Rule for Time-Delayed Systems}

To obtain a linear adaptation rule, the MIT rule, which leads to nonlinearity, is changed to classical PID controller. ML-MR PID-PID-PFA control is implemented, as illustrated in Figure 16.

The design steps of the ML-MR PID-PID-PFA control structure for a first order time delay plant in the form of $G(s)=\frac{K}{\tau s+1} e^{-L s}$ can be carried out as follows.

Step 1: Identify the model of the plant function and take the reference model of the MRC loop as the plant function $\left(T_{m}(s)=G(s)\right)$.

Step 2: Enclose the MRC loop with the optimal closed PID controller loop, as shown in Figure 16. Tavakoli-Tavakoli PID tuning rule, which is expressed by Equation (10), is used for the both PID controller functions. 


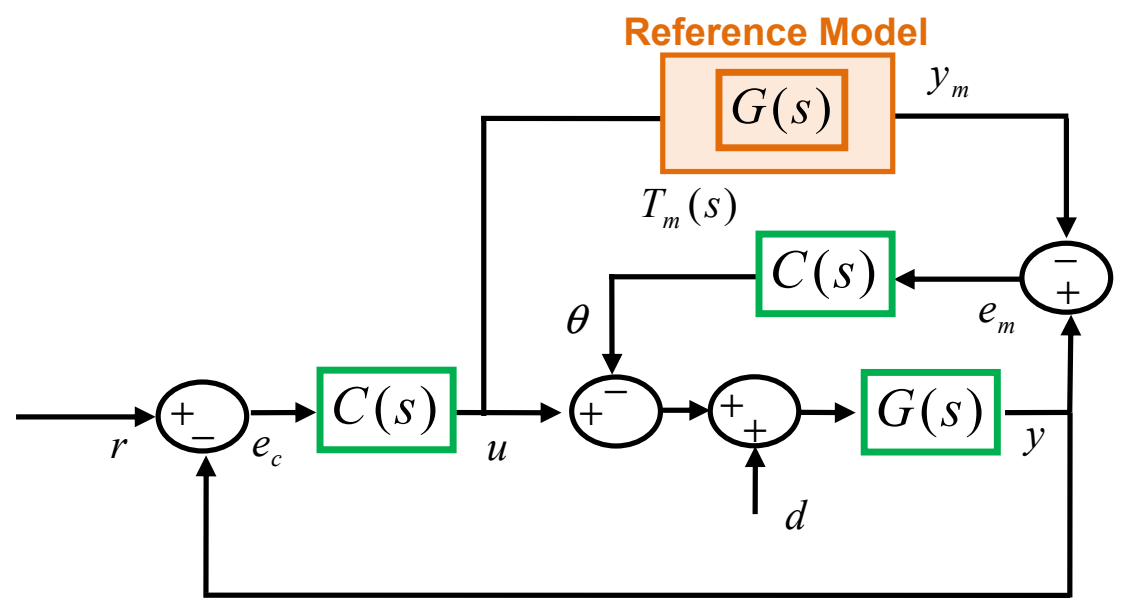

Figure 16. Block diagram of ML-MR PID-PID-PFA control structure.

To demonstrate the effectiveness of the ML-MR MIT-PID-PFA and ML-MR PID-PID-PFA control structures, we used them to enhance the first order plus deadtime plant model, which was given by Monje et al. [34].

$$
G(s)=\frac{3.13}{433.33 s+1} e^{-50 s}
$$

This plant function is a first order dynamical model of the Basic Process Rig 38-100 Feedback Unit (produced by Feedback Instruments Ltd) experimental platform [34]. This system presents a $50 \mathrm{~s}$ time delay with a time constant of $433.33 \mathrm{~s}$ and is considered a large time delay process. Monje et al. designed an optimal fractional order PID (FOPID) controller for this system as follows:

$$
C_{\text {FOPID }}(s)=0.6152+\frac{0.01}{s^{0.8968}}+4.3867 s^{0.4773}
$$

We implemented the ML-MR MIT-PID-PFA and ML-MR PID-PID-PFA control structures for this system by following the design steps given above. For control performance comparison purposes, we implemented a classical optimal PID control system with the Tavakoli-Tavakoli PID tuning rule $\left(k_{p}=1.1862, k_{i}=0.0026, k_{d}=22.8885\right)$, a classical optimal PID control loop with MATLAB optimal tuning $\left(k_{p}=0.8029, k_{i}=0.002, k_{d}=-35.3930\right)$, and an optimal FOPID control system with Monje et al.'s tuning rule. The set-point of this experimental system was originally 0.47 [34] and a step disturbance with an amplitude of 0.47 at $1000 \mathrm{~s}$ was applied to the input of the plant model. The results of the control system simulations are shown in Figure 17. The simulation results reveal that both the set-point and disturbance rejection performances of the ML-MR PID-PID-PFA structure re better than other controllers. The lowest overshoot and fastest settling results were achieved by the ML-MR PID-PID-PFA structure. It is noteworthy to observe that the control performance of the ML-MR PID-PID-PFA structure is superior to the optimal FOPID control system. This finding is a clear indication of the potential for multi-loop PID controllers to surpass the control performance of the FOPID controllers. The FOPID controllers have been considered as substitutes for classical PID control and have been extensively studied in the last decade, showing performance improvements of FOPID controllers over classical PID controllers. A major complication in practice for FOPID controllers is their realization complexity. Fractional order controllers can be implemented by using integer order models because near-ideal realization of fractional order derivatives and integral elements in digital systems is highly computationally expensive [2]. Recently, analog circuit realizations of fractional order controllers have been shown, with results promising low-cost and low-complexity solutions for practical realization of FOPID controllers $[35,36]$. Recent works have shown the robust control performance improvement of MR-ML control structures with FOPID controllers [25,26], indicating an avenue for robust control system research. 


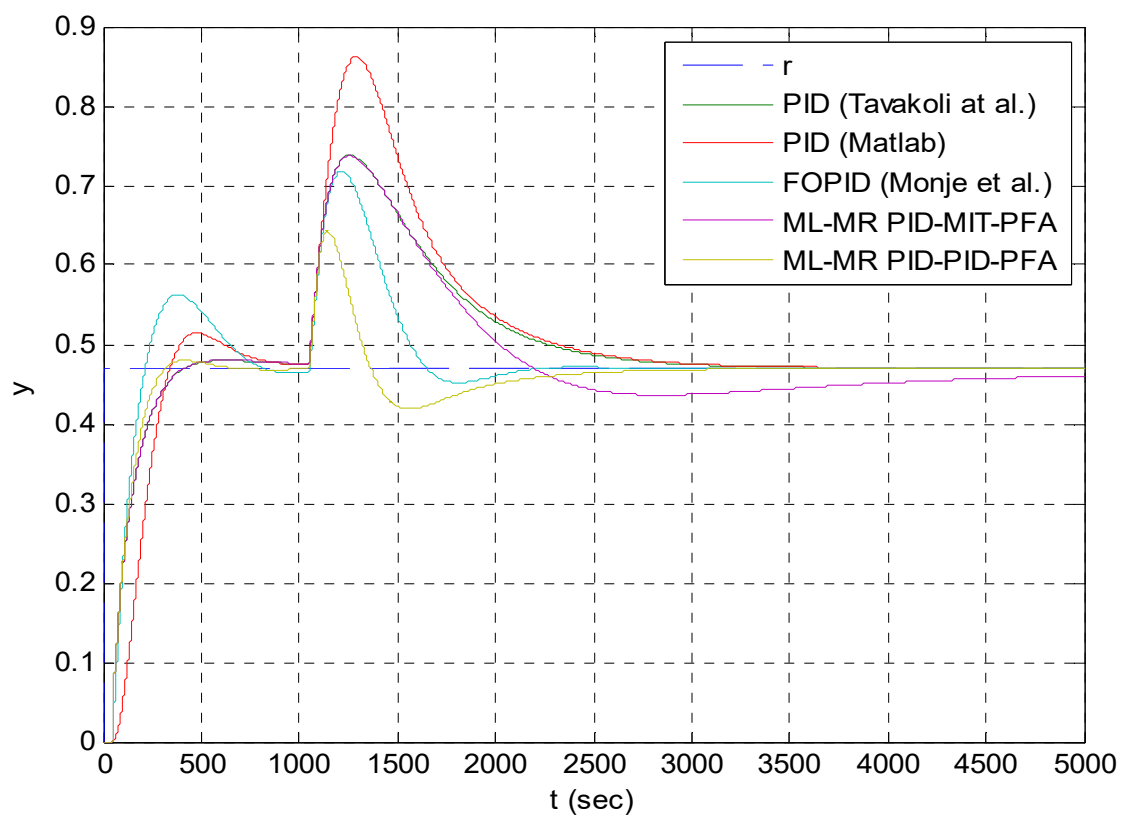

Figure 17. Control performance of various control systems for robust performance optimal control of a large time delay plant model [34].

It is useful to investigate the frequency dependence of disturbance rejection performance. For this purpose, the performances of the classical closed loop PID control structure and ML-MR PID-PID-PFA control structure were compared in the control problem of the Basic Process Rig 38-100 Feedback Unit experimental platform [34] using sinusoidal waveform disturbance signals at several frequencies. The PID controller was tuned according to the ITAE design rule proposed by Tavakoli et al. Table 1 shows the mean absolute error (MAE) of the control error signal $\left(\frac{1}{T} \int_{0}^{T}\left|e_{\mathcal{C}}(t)\right| d t\right)$ from simulations. For step disturbance, the ML-MR control structure reduces 50\% the MAE of classical PID control system. In general, environmental disturbances are altered slowly and involve low frequency components. For very low frequency disturbance signals, the MAE of the ML-MR PID-PID-PFA control structure decreases down to $25 \%$ of the MAE of the classical PID control system. As the disturbance frequency increases, the MAE performance of the ML-MR PID-PID-PFA control structure equals the classical PID control structure. Figure 18 shows some of the simulation results and confirms these findings.

Table 1. Mean absolute error (MAE) of control error signals obtained from control simulations for step and sinusoidal waveform disturbances.

\begin{tabular}{cccccccc}
\hline $\begin{array}{c}\text { Angular Frequencies } \\
\omega(\mathrm{rad} / \mathrm{s})\end{array}$ & $\begin{array}{c}\text { Step } \\
\text { Disturbance }\end{array}$ & 0.001 & 0.005 & 0.01 & 0.05 & 0.1 & 0.5 \\
\hline $\begin{array}{c}\text { Classical PID Control } \\
\text { (Tavakoli et al.) }\end{array}$ & 0.0470 & 0.0868 & 0.1748 & 0.1605 & 0.0560 & 0.0267 & 0.0145 \\
\hline ML-MR PID-PID-PFA & 0.0234 & 0.0216 & 0.1037 & 0.1465 & 0.0690 & 0.0250 & 0.0140 \\
\hline
\end{tabular}



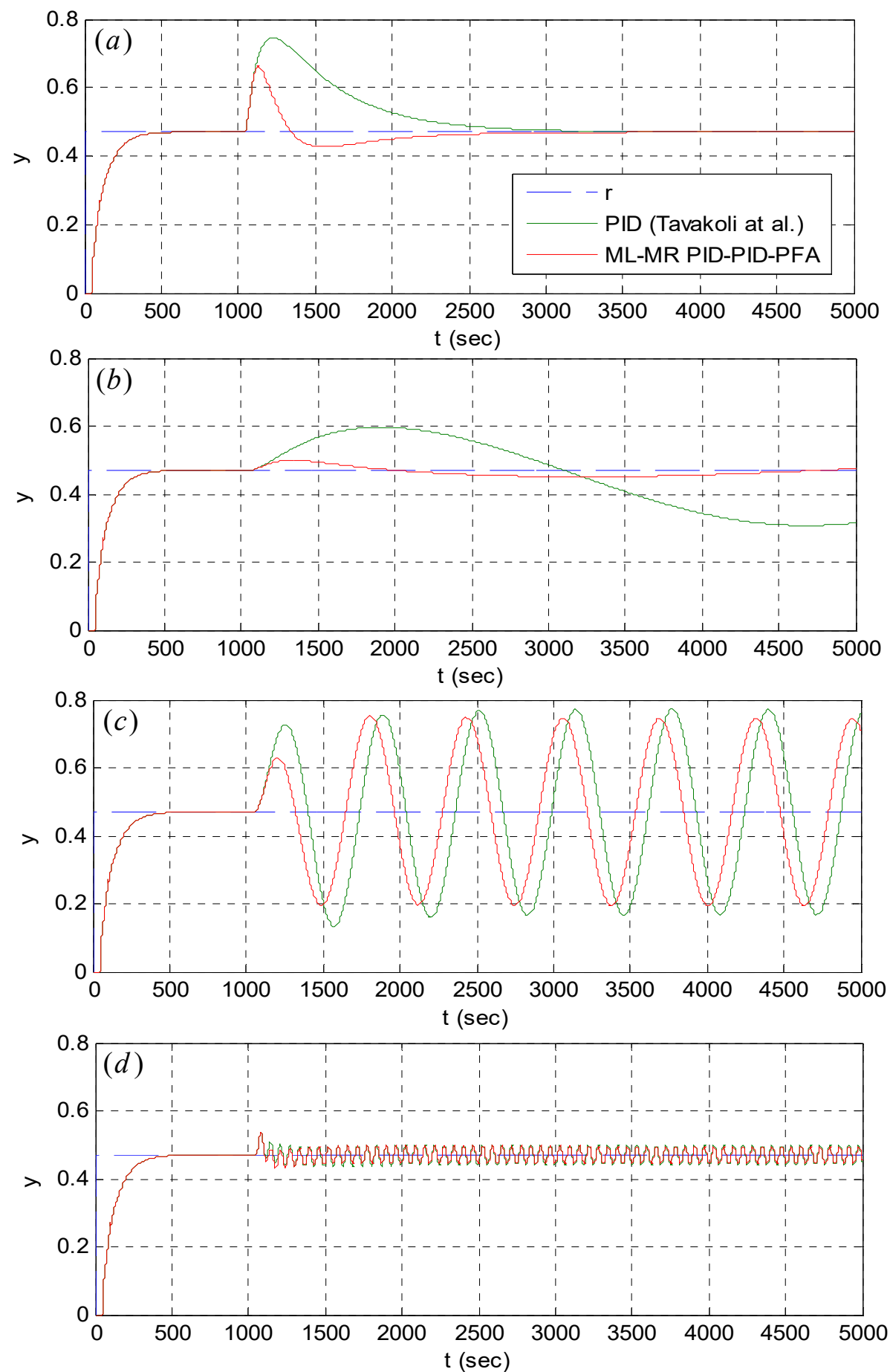

Figure 18. Comparisons of disturbance rejection performances of classical PID control and ML-MR PID-PID-PFA control structures for (a) step disturbance, (b) sinusoidal disturbance with a frequency of $0.001 \mathrm{rad} / \mathrm{s},(\mathbf{c})$ sinusoidal disturbance with a frequency of $0.01 \mathrm{rad} / \mathrm{s}$, and (d) sinusoidal disturbance with a frequency of $0.1 \mathrm{rad} / \mathrm{s}$.

In former studies, a ML-MR control structure using the MIT rule was experimentally verified for control of an experimental magnetic levitation system [26] and control of an experimental electrical rotor [28]. We conducted an experimental study to validate the contribution of the ML-MR PID-PID-PFA control structure to the disturbance rejection performance of classical PID control systems. A twin-rotor multi-input multi-output (MIMO) system (TRMS) is a popular rotor control test platform that is preferred for electrical rotor control experiments $[37,38]$. The TRMS is composed of two electrical 
rotors [39]. These are pitch and yaw rotors, as illustrated in Figure 19. The angles of the rotors are controlled by regulating the input voltages of the DC electric motors. This adjusts the rotational speed of the propellers so that the rotors can hover.

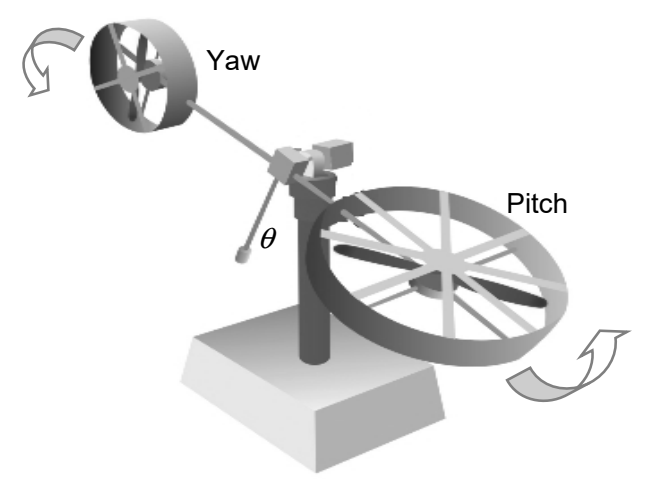

Figure 19. Experimental twin-rotor multi-input multi-output system (TRMS) setup [39].

Figure 20 shows the experimental results. After settling the pitch angle to $0.5 \mathrm{rad}$, an input disturbance with a unit step waveform was applied at $25 \mathrm{~s}$. One can observe that the step-point control performances of the classical PID controller and ML-MR PID-PID-PFA control structure are almost the same. The slight differences are mainly caused by internal system noise and limitations of the reference model in representing whole dynamics in the main rotor of the TRMS experimental setup. When disturbance is applied to the system, the response of ML-MR PID-PID-PFA control structure differs from the response of the PID controller and better rejects the disturbance at the system output, as shown in the figure.

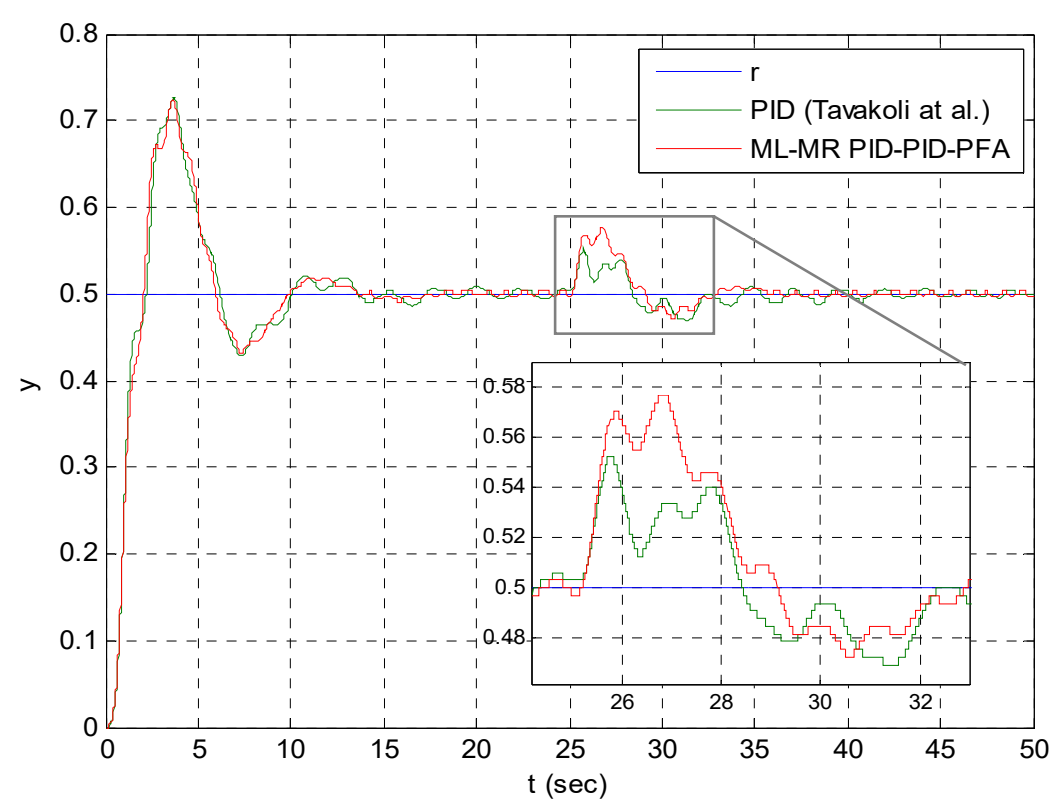

Figure 20. Experimental results obtained for control of the main rotor of TRMS setup.

One of major technical complications that was observed in the experimental study was the model mismatch problem in the real system. We used a nonlinear model of TRMS that is a good representation of experimental systems. However, inherent limitations of mathematical modeling and changes of operating conditions reduce the model consistency and lead to model mismatching problems. Since the response of reference model does not adequately match with the response of the real TRMS setup, the PID controller of the adaptation loop, which generates the adaptation gain $\theta$, can cause instability 
in the system for the same PID controller coefficients for the control loop. This stability problem can be solved by retuning the PID controller to the adaptation loop.

\section{Discussions and Conclusions}

This study investigated some contemporary ML-MR adaptive control structures that are applicable for classical PID control loops. These structures are the ML-MR PID-MIT, ML-MR PID-MIT-CGM, ML-MR PID-I, ML-MR PID-IM, ML-MR MIT-PID-PFA, and ML-MR PID-PID-PFA control structures. Design problems of these ML-MR structures were addressed, and straightforward design and implementation strategies were presented. The performance evaluations, advantages, and drawbacks of these ML-MR structures were discussed according to control simulation results. Simulation results indicated significant disturbance rejection performance improvements in four different control application examples. The performance comparisons with several single-loop optimal control loops theoretically show that improvement of the disturbance rejection control without affecting the set-point performance is possible with presented ML-MR control structures. Some noteworthy properties of the presented ML-MR adaptive control structures are listed in Table 2.

Table 2. Some properties of the presented ML-MR control structures.

\begin{tabular}{cccccc}
$\begin{array}{c}\text { ML-MR Control } \\
\text { Structures }\end{array}$ & Character & $\begin{array}{c}\text { Dependence to } \\
\text { Set-Point } \\
\text { Level }\end{array}$ & $\begin{array}{c}\text { Negative Effects } \\
\text { on Set-Point } \\
\text { Performance }\end{array}$ & $\begin{array}{c}\text { Applicability for Time } \\
\text { Delay Systems }\end{array}$ & $\begin{array}{c}\text { Indications in } \\
\text { Simulation } \\
\text { Results }\end{array}$ \\
\hline $\begin{array}{c}\text { ML-MR PID-MIT } \\
\text { ML-MR }\end{array}$ & Nonlinear & Dependent & None & None & Fone \\
PID-MIT-CGM & Nonlinear & Dependent & None 3 and 4 \\
ML-MR PID-I & Linear & Independent & None & None & None \\
ML-MR PID-IM & Linear & Independent & None & Appligure 10 \\
ML-MR & Nonlinear & Dependent & None to some degree & Figure 17 \\
MIT-PID-PFA & Linear & Independent & None & Applicable to some degree \\
ML-MR & & Figure 17 &
\end{tabular}

Some notable remarks: * ML-MR adaptive control structures are applicable in all classical control loops in order to improve the disturbance rejection performance without modifying the parameters of the existing control system or degrading the set-point performance. This is an important advantage of hierarchical ML-MR structures in control engineering. * Integration approaches of the model reference control loop into classical control loops are carried out in two steps: outer loop integration uses closed loop control systems as reference models, such as ML-MR PID-MIT, ML-MR PID-MIT-CGM, ML-MR PID-I, and ML-MR PID-IM controls; and inner loop integration uses models of plant or process functions as reference models, such as ML-MR MIT-PID-PFA and ML-MR PID-PID-PFA. Outer loop integrations cover the whole closed control loop to deal with disturbances, and several works have indicated that these type integrations are preferable solutions for improving fault tolerance and disturbance rejection in the whole control loop [25-28]. Inner loop integration only encloses the plant function, and hence it can be employed to deal with external disturbances or parametric perturbations that influence the controlled system. ${ }^{*} \mathrm{ML}-\mathrm{MR}$ PID-MIT-CGM and ML-MR PID-I structures can adaptively increase the RDR performance of the closed control loops without affecting set-point performance. The ML-MR PID-IM control structure benefits from having an internal model controller design, however it should be noted that its performance is very sensitive to the accuracy of plant and controller models. ${ }^{*}$ Large-time delay systems that set the point and disturbance rejection performance of ML-MR PID-PID-PFA control structures can surpass the disturbance rejection performance of a single-loop optimal FOPID control system. These results reveal that the disturbance rejection capacity of closed loop PID control loops can be exceeded by combining them with ML-MR control structures. ML-MR control structures are a feasible way to resolve performance tradeoff between set-point and disturbance rejection control structures. * Stability analysis of linear ML-MR control structures is relatively straightforward and is performed by obtaining the transfer function of the whole structure and checking for pole placements for left half-plane (LHP) stability. The satiability of a nonlinear ML-MR structure, such as ML-MR PID-MIT control, along with the stability and convergence conditions, are derived on the basis of the bounded input and bounded output stability state of the model error (see Appendix A). * Results in this study are based on simulation models. The reference models perfectly match the controlled systems. It is very important to note that model mismatches due to limitations in mathematical modeling and parametric model perturbations in real systems may have severe effects on the control performance and stability of the presented ML-MR adaptive control structures in real-world applications. The sensitivity of these structures to model mismatching should be carefully investigated in future works. The fragility of the system stability due to model imprecision should be carefully considered when designing real control systems. Moreover, the resolution of sensors and I/O ports of control cards are important in practical applications. ${ }^{*}$ This study is a theoretical demonstration of six different ML-MR adaptive control structures. Future works should address experimental performance evaluations and practical realization issues of ML-MR adaptive control structures. 
The most prominent contributions of the introduced hierarchical ML-MR control structures are:

(i) The proposed ML-MR adaptive control structures can be applied to existing control loops without modifying any parameters of the control loops. These structures do not require online return of the control loop, which may result in instant performance degradation while altering controller coefficients. Therefore, ML-MR control structures provide more consistent control performance than conventional MRAC structures that are used to perform online return of the control loop.

(ii) The proposed ML-MR adaptive control structures increase the disturbance rejection performance without deteriorating the set-point control quality. This is an important contribution to the solution of performance tradeoffs between disturbance rejection and set-point control.

Author Contributions: Conceptualization, B.B.A. and A.T.; methodology, B.B.A. and A.T.; validation, E.P. and C.Y.; writing - original draft preparation, B.B.A.; writing-review and editing, A.T., E.P., and C.Y.; supervision, E.P. and C.Y. All authors have read and agreed to the published version of the manuscript.

Funding: This research was funded by the European Cooperation in Science and Technology (COST) Action CA15225. The work of Eduard Petlenkov and Aleksei Tepljakov was supported by the Estonian Research Council grant PRG658.

Acknowledgments: This article is based upon work from COST Action CA15225, a network supported by the European Cooperation in Science and Technology (COST). The work of Eduard Petlenkov and Aleksei Tepljakov was supported by the Estonian Research Council grant PRG658.

Conflicts of Interest: The authors declare no conflict of interest. The funders had no role in the design of the study; in the collection, analyses, or interpretation of data; in the writing of the manuscript, or in the decision to publish the results.

\section{Appendix A}

Since the inner PID control loop is designed as a stable system, convergence of the model error $e_{m}=y-y_{m}<\varepsilon \in R$ ensures the bounded input and bounded output stability of the ML-MR PID-MIT control structure. In this manner, we can investigate the convergence condition of $e_{m}$.

Lemma A1. For the ML-MR PID-MIT control structure, the model error becomes zero $\left(e_{m}=0\right)$ when the adaptation gain $\theta$ is equal to 1 and $T(s)=T_{m}(s)$.

Proof A1. Let us consider model error $e_{m}=y-y_{m}=T(s) \theta r-T_{m}(s) r$ for the ML-MR PID-MIT control structure and apply the condition of $\theta=1$ and $T(s)=T_{m}(s)$. Accordingly, one can show that:

$$
e_{m}=T(s) \theta r-T_{m}(s) r=T_{m}(s) r-T_{m}(s) r=0
$$

Theorem A1 (Stability of the ML-MR PID-MIT Control Structure). For a stable system model $T(s)=$ $T_{m}(s)$, the model of the ML-MR PID-MIT control structure is stable only if the model error function is as follow:

$$
e_{m}=-\frac{1}{\gamma}\left(s^{2}+\frac{s^{2}}{C(s) G(s)}\right)
$$

where the bounded input and bounded output are stable. For the below equation the model error converges to zero.

$$
\lim _{s \rightarrow 0^{+}}\left(s^{3}+\frac{s^{3}}{C(s) G(s)}\right)=0
$$


Proof A2. Let us consider the adaptation rule of the ML-MR PID-MIT control structure, which is given by Equation (9) as $\theta=-\gamma \frac{1}{s} y_{m} e_{m}$. The model can be written as:

$$
e_{m}=\frac{s \theta}{-\gamma y_{m}}
$$

The output of the reference model $y_{m}$ can be written as $y_{m}=T_{m}(s) r(s)$. For set-point control stability, one considers the step response of the system. Therefore, the reference input is assumed to be a step function by using $r(s)=\frac{1}{s}$ in Equation (A3).

$$
e_{m}=\frac{s^{2} \theta}{-\gamma T_{m}(s)}
$$

According to Lemma 1, the convergence of the model error to zero $\left(e_{m}=0\right)$ is possible for $\theta=1$ in the case of $T_{m}(s)=T(s)$. By applying this condition, the model error is written as:

$$
e_{m}=\frac{s^{2}}{-\gamma T(s)}
$$

The function $T(s)$ is the transfer function of the inner loop, which is stable due to optimal tuning of the PID control system. When the T(s) function given by Equation (2) is used in Equation (A5), one obtains

$$
e_{m}=-\frac{1}{\gamma}\left(s^{2}+\frac{s^{2}}{C(s) G(s)}\right)
$$

The inner loop is configured to be stable, therefore the output of the inner loop is bounded for $y<\xi \in R$ for a step reference input. When the model error $e_{m}$ of the system given by Equation (A6) is shown to be stable, the bounded input and bounded output of the ML-MR PID-MIT control structure become stable, so that $e_{m}=y-y_{m}<\varepsilon \in R$ is satisfied. In order to ensure convergence of the model error to zero, a final value theorem can be applied as:

$$
\lim _{s \rightarrow 0^{+}} s e_{m}=0
$$

Then, one obtains the convergence condition as follows:

$$
\lim _{s \rightarrow 0^{+}}\left(s^{3}+\frac{s^{3}}{C(s) G(s)}\right)=0 .
$$

\section{References}

1. Fossard, A.J.; Normand-Cyrot, D. Nonlinear Systems Modeling and Estimation; Springer: Berlin, Germany, 1995.

2. Tepljakov, A. Fractional-Order Modeling and Control of Dynamic Systems; Springer: Berlin, Germany, 2017.

3. Lanchier, N. Stochastic Modeling; Springer: Berlin, Germany, 2017.

4. Alagoz, B.B.; Tan, N.; Deniz, F.N.; Keles, C. Implicit disturbance rejection performance analysis of closed loop control systems according to communication channel limitations. IET Control Theory A 2015, 9, 2522-2531. [CrossRef]

5. Butler, H.; Honderd, G.; Van Amerongen, J. Model reference adaptive control of a direct-drive DC motor. IEEE Control Syst. Mag. 1989, 9, 80-84. [CrossRef]

6. Barkana, I.; Kaufman, H.; Balas, M. Model reference adaptive control of large structural systems. J. Guid. Control Dyn. 1983, 6, 112-118. [CrossRef]

7. Duarte, M.A.; Narendra, K.S. A new approach to model reference adaptive control. Int. J. Adapt. Control. 1989, 3, 53-73. [CrossRef] 
8. Barkana, I. Simple adaptive control-a stable direct model reference adaptive control methodology-brief survey. Int. J. Adapt. Control 2014, 28, 567-603. [CrossRef]

9. Yamamoto, T.; Takao, K.; Yamada, T. Design of a data-driven PID controller. IEEE Trans. Control Syst. Technol. 2008, 17, 29-39. [CrossRef]

10. Barbosa, R.S.; Machado, J.T.; Ferreira, I.M. Tuning of PID controllers based on Bode's ideal transfer function. Nonlinear Dyn. 2004, 38, 305-321. [CrossRef]

11. Wellenreuther, A.; Gambier, A. Multi-loop Controller Design for a Heat Exchanger. In Proceedings of the 2006 IEEE International Conference on Control Applications, Munich, Germany, 4-8 October 2006; pp. 2099-2104.

12. Vijayan, V.; Panda, R.C. Design of PID controllers in double feedback loops for SISO systems with set-point filters. ISA Trans. 2012, 51, 514-521. [CrossRef]

13. Lee, M.; Lee, K.; Kim, C.; Lee, J. Analytical Design of Multiloop PID Controllers for Desired Closed-Loop Responses. AIChE J. 2004, 50, 1631-1635. [CrossRef]

14. Shamsuzzoha, M.; Lee, M. IMC-PID Controller Design for Improved Disturbance Rejection of Time-Delayed Processes. Ind. Eng. Chem. Res. 2007, 46, 2077-2091. [CrossRef]

15. Visioli, A. A new design for a PID plus feedforward controller. J. Process Control 2004, 14, 457-463. [CrossRef]

16. Su, W.A. Model Reference-Based Adaptive PID Controller for Robot Motion Control of Not Explicitly Known Systems. IJICS 2007, 12, 237-244.

17. Trajkov, T.N.; Köppe, H.; Gabbert, U. Direct model reference adaptive control (MRAC) design and simulation for the vibration suppression of piezoelectric smart structures. Commun. Nonlinear Sci. 2008, 13, 1896-1909. [CrossRef]

18. Landau, I.D. Adaptive Control the Model Reference Approach; Marcel Dekker: New York, NY, USA, 1979.

19. Astrom, K.J.; Wittenmark, B. Adaptive Control; Addison-Wesley: Boston, MA, USA, 1995.

20. Butler, H. Model-Reference Adaptive Control-From Theory to Practice; Prentice-Hall: Upper Saddle River, NJ, USA, 1992.

21. Vinagre, B.M.; Petrás, I.; Podlubny, I.; Chen, Y.Q. Using fractional order adjustment rules and fractional order reference models in model-reference adaptive control. Nonlinear Dyn. 2001, 29, 269-279. [CrossRef]

22. Kavuran, G.; Alagoz, B.B.; Ates, A.; Yeroglu, C. Implementation of model reference adaptive controller with fractional order adjustment rules for coaxial rotor control test system. BAJECE 2016, 4, 84-88.

23. Kavuran, G.; Ates, A.; Alagoz, B.B.; Yeroglu, C. An Experimental Study on Model Reference Adaptive Control of TRMS by Error-Modified Fractional Order MIT Rule. Control Eng. Appl. Inf. 2017, 19, 101-111.

24. Benaskeur, A.R.; Desbiens, A. Backstepping-based adaptive PID control. IET Control Theory A 2002, 149, 54-59.

25. Alagoz, B.B.; Tepljakov, A.; Petlenkov, E.; Yeroglu, C. Multi-Loop model reference adaptive control of fractional-order PID control systems. In Proceedings of the 2017 40th International Conference on Telecommunications and Signal Processing (TSP), Barcelona, Spain, 5-7 July 2017; pp. 702-705.

26. Tepljakov, A.; Alagoz, B.B.; Gonzalez, E.; Petlenkov, E.; Yeroglu, C. Model reference adaptive control scheme for retuning method-based fractional-order PID control with disturbance rejection applied to closed-loop control of a magnetic levitation system. J. Circuits Syst. Comput. 2018, 27, 1850176. [CrossRef]

27. Rajesh, R.; Deepa, S.N. Design of direct MRAC augmented with 2 DoF PIDD controller: An application to speed control of a servo plant. J. King Saud Univ. Eng. Sci. 2019. [CrossRef]

28. Alagoz, B.B.; Kavuran, G.; Ateş, A.; Yeroğlu, C.; Alisoy, H. Multi-loop Model Reference Adaptive PID Control for Fault-Tolerance. BAJECE 2019, 7, 276-285. [CrossRef]

29. Tavakoli, S.; Tavakoli, M. Optimal tuning of PID controllers for first order plus time delay models using dimensional analysis. In Proceedings of the 2003 4th International Conference on Control and Automation Proceedings, Montreal, QC, Canada, 12 June 2003; pp. 942-946.

30. Alagoz, B.B.; Deniz, F.N.; Keles, C.; Tan, N. Disturbance rejection performance analyses of closed loop control systems by reference to disturbance ratio. ISA Trans. 2015, 55, 63-71. [CrossRef] [PubMed]

31. Bendjeghaba, O.; Boushaki, S.I. Optimal Tuning of PID Controller in Automatic Voltage Regulator System using Improved Harmony Search Algorithm. In Proceedings of the 7th Global Conference on Power Control and Optimization, Prague, Czech, 25-27 August 2013; pp. 1-6.

32. Zhang, P. Industrial control system simulation routines. In Advanced Industrial Control Technology; Elsevier: Oxford, UK, 2010; pp. 781-810. 
33. Chen, D.; Seborg, D.E. PI/PID Controller Design Based on Direct Synthesis and Disturbance Rejection. Ind. Eng. Chem. Res. 2002, 41, 4807-4822. [CrossRef]

34. Monje, C.A.; Vinagre, B.M.; Feliu, V.; Chen, Y.Q. Tuning and auto-tuning of fractional order controllers for industry applications. Control Eng. Pract. 2008, 16, 798-812. [CrossRef]

35. Muñiz-Montero, C.; García-Jiménez, L.V.; Sánchez-Gaspariano, L.A.; Sánchez-López, C.; González-Díaz, V.R.; Tlelo-Cuautle, E. New alternatives for analog implementation of fractional-order integrators, differentiators and PID controllers based on integer-order integrators. Nonlinear Dyn. 2017, 90, 241-256. [CrossRef]

36. Domansky, O.; Sotner, R.; Langhammer, L.; Jerabek, J.; Psychalinos, C.; Tsirimokou, G. Practical design of RC approximants of constant phase elements and their implementation in fractional-order PID regulators using CMOS voltage differencing current conveyors. Circuit Syst. Signal Process. 2019, 38, 1520-1546. [CrossRef]

37. Alagoz, B.B.; Ates, A.; Yeroglu, C. Auto-tuning of PID controller according to fractional-order reference model approximation for DC rotor control. Mechatronics 2013, 23, 789-797. [CrossRef]

38. Rahideh, A.; Shaheed, M.H. Constrained output feedback model predictive control for nonlinear systems. Control Eng. Pract. 2012, 20, 431-443. [CrossRef]

39. Twin Rotor MIMO System Control Experiments 33-949S; Feedback Instruments Ltd.: East Sussex, UK.

(C) 2020 by the authors. Licensee MDPI, Basel, Switzerland. This article is an open access article distributed under the terms and conditions of the Creative Commons Attribution (CC BY) license (http://creativecommons.org/licenses/by/4.0/). 